COMMUNICATIONS IN

ANALYSIS AND GEOMETRY

Volume 4, Number 1, 129-160, 1996

\title{
Examples of non-positively curved Kähler manifolds ${ }^{1}$
}

\author{
FANGYANG ZHENG ${ }^{2}$
}

\section{Introduction.}

Let $\mathcal{N}_{n}(n \geq 2)$ be the set of $n$-dimensional compact complex manifolds which admit Kähler metrics with quasi-negative sectional curvature (i.e., non-positive everywhere and negative somewhere). Despite the general belief that such manifolds should be abundant, there are very few known examples constructed. In fact, before 1980, the only known such examples are the locally hermitian symmetric ones. In 1980, Mostow and Siu ([M-S]) constructed a sequence of negatively curved surfaces which are not locally symmetric. This sequence of examples are still the only known manifolds in $\mathcal{N}_{n}$, other than the locally symmetric ones, for any $n \geq 2$.

In this paper, we shall construct three new sequences of surfaces in $\mathcal{N}_{2}$. The idea is very simple, one starts with a compact complex ball quotient $X^{n}$ which contains a totally geodesic divisor $D$. If $Y$ is a finite branched covering of $X$ with ramifications $R$ over $D$ (of simple type), then one can add a suitably choosing metric, which supports in a neighborhood of the divisor $R$, to the pull-back of the canonical metric of $X$. The sum metric is then quasi-negatively curved. When $n=2$, there are such surfaces $Y$ constructed by Hirzebruch in his famous paper on the arrangement of lines $([\mathrm{H}])$. These surfaces are different from the ones constructed by Mostow and Siu, since they have different ratios of the two Chern numbers (cf. §3).

For $n>2$, the same constructions should also give manifolds in $\mathcal{N}_{n}$, if we knew examples of such $Y^{n}$ over such a pair $\left(X^{n}, D\right)$ of compact ball quotient and totally geodesic divisor $D$ in it.

In $\S 4$, we also discuss the finite volume case. Theorem 3 says that if $X^{n}$ is a smooth compact complex ball quotient and $D$ is a smooth totally geodesic

\footnotetext{
${ }^{1} 1980$ Mathematics Subject Classification (1985 Revision). Primary 53C55.

${ }^{2}$ Research partially supported by a NSF Grant and a Sloan Foundation Fellowship.
} 
divisor in $X$. Then the complement $Z=X \backslash D$ admits complete Kähler metric with finite volume whose complex curvature operator is negative and bounded from below. As a consequence, the complex structure on any of these three families of surfaces of line arrangements is unique.

For any $n \geq 2$, the existence of such a pair $\left(X^{n}, D\right)$ is proved by Kudla and Millson (cf. p.129-130 of [K-M]). So one has negatively curved complete Kähler manifolds of finite volume in any dimensions.

The main technical contribution of this paper is the proof of Theorem 2 in $\S 2$, where we need to compute the curvature of the sum of two metrics. It is technically much more difficult than the smooth divisor case of Theorem 1. In this regards, we should mention the work of Cheung and $\mathrm{Wu}([\mathrm{C}-\mathrm{W}])$, where they initiated the systematic study of the sum of two complex hyperbolic type metrics.

In the Riemannian case, in 1987, Gromov and Thurston constructed in [G-T] many examples of negatively curved Riemannian manifolds in all dimensions. Their starting point was a compact manifold $X$ with constant negative curvature which contains a (real) codimension two totally geodesic submanifold $D$, and they perform geometric surgery on a cyclic cover $Y$ of $X$ which is branched along $D$. So this paper can be regarded as a complex analogue of their work, although the way to prove the negativity of the curvature is totally different.

Acknowledgements. We would like to thank mathematicians Pat Eberlein, John Millson, Mark Stern, Gang Tian and S-T Yau for valuable suggestions, helps, and encouragement.

\section{The metric.}

First of all, we need the following terminology

Definition. Let $f: Y \rightarrow X$ be a surjective holomorphic map between two compact complex manifolds of dimension $n$. $Y$ will be called a good covering over $X$, if for any $p \in Y$, there exits holomorphic coordinates $\left\{z_{1}, \ldots, z_{n}\right\}$ near $p$ and $\left\{w_{1}, \ldots, w_{n}\right\}$ near $f(p)$ such that $p=(0, \ldots, 0)$ and in a neighborhood of $p, f$ is given by $w_{i}=z_{i}^{m_{i}}, i=1, \ldots, n$, where $m_{i}$ are positive integers.

Note that for a good covering $f: Y \rightarrow X$, the branching locus $D$ is a (locally) normal crossing divisor (the components may have self-intersections). 
Next, let $X^{n}$ be a compact smooth quotient of the complex $n$-ball $\mathbf{B}^{n} \subseteq$ $\mathbf{C}^{n}$, equipped with the canonical metric $g$. Let $D$ be a smooth totally geodesic divisor in $X$. Let $\rho=\rho_{D}$ be the distance from $D$. It is a smooth function in $U \backslash D$ for some tubular neighborhood $U$ of $D$.

Definition. For $X, D$ and $\rho$ as above, the function $d=d_{D}=\left(\frac{e^{\rho}-1}{e^{\rho}+1}\right)^{2}$ will be called the Poincare (square) distance from the divisor $D$. It is a smooth function in a tubular neighborhood $U$ of $D$.

Note that for the $n$-ball $\mathbf{B}^{n}=\left\{\left|z_{1}\right|^{2}+\cdots+\left|z_{n}\right|^{2}<1\right\}$ and the totally geodesic divisor $D=\left\{z_{1}=0\right\}$, the Poincare distance takes the simple form $d_{1}(z)=\frac{\left|z_{1}\right|^{2}}{1-\left|z_{2}\right|^{2}-\cdots-\left|z_{n}\right|^{2}}$.

Next, suppose $f: Y^{n} \rightarrow X^{n}$ is a good covering over a ball quotient $X$ with totally geodesic branching locus $D$. Let $g$ be the canonical metric on $X$ and $\omega_{g}$ its Kähler form. Let $R=\left\{p \in Y \mid \operatorname{det}\left(d f_{p}\right)=0\right\}$ be the ramification locus. Since the metric $f^{*} g$ degenerate along $R$, we want to define a correction metric near $R$ and add it to $f^{*} g$.

For a sufficiently small $\delta>0$, let $U=U_{\delta}$ be a $\delta$-neighborhood of $D$ and let $V$ be the components of $f^{-1}(U)$ that contains $R$. For any $p \in R$, choose holomorphic coordinates $\left\{z_{1}, \cdots, z_{n}\right\}$ centered at $p$ and $\left\{w_{1}, \cdots, w_{n}\right\}$ centered at $f(p)$ such that $f$ is given by $w_{i}=z_{i}^{m_{i}}$ for each $i$. Let us assume that $m_{i}>1$ for $1 \leq i \leq r$, and $m_{i}=1$ for $r<i \leq n$. Write $D_{i}=\left\{w_{i}=0\right\}, 1 \leq i \leq r$, and let $d_{i}$ be the Poincare distance function from the divisor $D_{i}$. Define

$$
\Phi=G_{m_{1}}\left(x_{1}\right) \cdots G_{m_{r}}\left(x_{r}\right) \cdot f^{*}\left(\omega_{g}^{n}\right)
$$

where

$$
G_{m}(x)=\frac{(1-\bar{m} x)\left(1-x^{m}\right)}{(1-x)^{3} x^{m-1}}, \quad \bar{m}=\frac{m-1}{m+1}
$$

and $x_{i}=f^{*}\left(\rho \circ d_{i}^{\frac{1}{m_{i}}}\right)$, with $\rho$ a smooth, non-decreasing cut-off function defined on $[0, \infty]$, such that $\rho(t) \equiv t$ near $t=0$ and $\rho \equiv c$ near and after $t=\delta$. Here $c$ is a small positive constant. It is not hard to check that $\Phi$ is well-defined, and is smooth and positive along $R$. So it is a volume form on $Y$.

Now the desired metric on $Y$ is just

$$
\omega_{\epsilon}=f^{*}\left(\omega_{g}\right)+\epsilon \sqrt{-1} \partial \bar{\partial} \log \Phi
$$

It is straight-forward to verify that $\omega_{\epsilon}$ is indeed everywhere positive-definite on $Y$ for all sufficiently small $\epsilon>0$. 
This correction term was first used by Mostow and Siu in [M-S] in the case of $n=2$ with $D$ smooth and on the universal covering level. In that special case it is just the Bergmann metric of the Thullen domain $\left\{\left|z_{1}\right|^{2 m}+\right.$ $\left.\left|z_{2}\right|^{2}<1\right\}$. We observe that it can be realized at downstairs by using the Poincare distance function.

The next section is devoted to the proof of the fact that the above constructed metric $\omega_{\epsilon}$ has quasi-negative complex curvature operator (hence strongly quasi-negatively curved in the sense of Siu). In fact the complex curvature operator is negative definite everywhere in $Y \backslash S$, where $S$ is the singular point set of $R$, which has codimension at least 2 .

\section{The curvature.}

First of all, we have the following result, the two dimensional case is due to Mostow and Siu in [M-S] (on the universal covering level).

Theorem 1. Let $f: Y^{n} \rightarrow X^{n}$ be a good covering over a smooth compact complex ball quotient $X^{n}$ and with totally geodesic branching divisor D. If $D$ is smooth, then $Y^{n}$ admits a Kähler metric with negative definite complex curvature operator.

In particular, $Y^{n}$ is strongly rigid (that is, any compact Kähler manifold homotopic equivalent to $Y$ must be (anti-)biholomorphic to $Y$ ).

Proof. The metric here is of course the one constructed in $\S 1$. It suffices to show that there exists $\epsilon_{0}>0$ and a neighborhood $V_{1}$ of $R$ such that $\omega_{\epsilon}$ has negative complex curvature operator in $V_{1}$ for any $0<\epsilon<\epsilon_{0}$. Note that it is important here that $V_{1}$ is independent of $\epsilon$. Then the compact complement $Y \backslash V_{1}$ can be taken care of by letting $\epsilon$ to be sufficiently small.

Fix a point $p \in V \backslash R$. Write $q=f(p)$ and let $q_{0}$ be the point on $D$ that is closest to $q$. Let $\left\{z_{1}, \cdots, z_{n}\right\}$ be a canonical coordinates of the unit ball $\mathbf{B}^{n}$ such that $q_{0}$ is (underneath) the origin and $D$ is (underneath) the hyperplane $\left\{z_{1}=0\right\}$. Then $q=(a, 0, \cdots, 0)$, and the Poincare distance function is $d=\frac{\left|z_{1}\right|^{2}}{1-\left|z_{2}\right|^{2}-\cdots-\left|z_{n}\right|^{2}}$.

Let $f_{1}$ be a local inverse map of $f$ which sends $q$ to $p$, and

$$
\varphi\left(z_{1}, \ldots, z_{n}\right)=\left(w, z_{2}, \ldots, z_{n}\right), \quad w=\frac{z_{1}+a}{1+\bar{a} z_{1}}
$$

The curvature of $\omega_{\epsilon}$ at $p$ is the same of the curvature of the metric

$$
\widetilde{\omega} \equiv\left(f_{1} \circ \varphi\right)^{*}\left(\omega_{\epsilon}\right)=\omega_{g}+\epsilon \omega_{h}=\omega_{g}+\epsilon \sqrt{-1} \partial \bar{\partial} h
$$


at the origin $q_{0}=0$, where

$$
h=\log \frac{v-\bar{m} t^{\frac{1}{m}}}{\left(v-t^{\frac{1}{m}}\right)^{3} v^{2 m-1}}
$$

and $v=(1-s)^{\frac{1}{m}}, t=|w|^{2}$ with $w=\frac{z_{1}+a}{1+\bar{a} z_{1}}, s=\left|z_{2}\right|^{2}+\cdots+\left|z_{n}\right|^{2}$. Here $m$ is the multiplicity of $R$ at $p$.

We ignored the cut-off function $\rho$ here since we are only interested in the situation very close to $R$.

By shifting some copies of $\omega_{g}$ into $\omega_{h}$ (this will result in a change of $\epsilon$ ) we may assume that the metric under consideration is

$$
\begin{gathered}
\widetilde{\omega}=\omega_{g}+\epsilon \omega_{h} \quad \text { with } \\
h=\log \left(\frac{v-\bar{m} t^{\frac{1}{m}}}{\left(v-t^{\frac{1}{m}}\right)^{3} v^{2 m-1}} \cdot \frac{1}{(1-t-s)^{4}}\right)
\end{gathered}
$$

(The reason of doing this shifting is to guarantee that $h$ is positive definite and is sufficiently negatively curved on the tangential directions of $D$, and we will still use the same notation for $\epsilon$ ).

At the point $q_{0}=0, t=|a|^{2}$ and $s=0$, hence $h_{i \bar{j}}=0$ for $i \neq j, h_{i \bar{j} k}=0$ except $h_{i \bar{i} 1}$, and all $h_{i \bar{j} k \bar{l}}=0$ except those $h_{i \bar{i} j \bar{j}}$. Since $g$ is normal at the origin, we have

$$
-R_{i \bar{j} k \bar{l}}=g_{i \bar{j} k \bar{l}}+\epsilon h_{i \bar{j} k \bar{l}}-\epsilon^{2} \sum_{p=1}^{n} h_{i \bar{p} k} h_{\bar{j} q \bar{l}}\left(g_{p \bar{p}}+\epsilon h_{p \bar{p}}\right)^{-1}
$$

Therefore, at $q_{0}$, all the components of $R_{i \bar{j} k \bar{l}}$ are zero except those $R_{i \bar{j} j \bar{j}}$. Denote it by $-a_{i j}$. Then the complex curvature operator of the considered metric will be negative definite at $q_{0}$ if and only if the matrix $A=\left(a_{i j}\right)$ is positive definite.

By the symmetry among $z_{2}, \ldots, z_{n}$, we know that the matrix $A$ takes the special form

$$
A=\left[\begin{array}{ccccc}
b & c & c & \cdots & c \\
c & d & e & \cdots & e \\
c & e & d & \cdots & e \\
\vdots & \vdots & \vdots & \ddots & \vdots \\
c & e & e & \cdots & d
\end{array}\right]
$$

where since

$$
-R_{i \bar{i} \bar{j}}=1+\delta_{i j}+\epsilon h_{i \bar{i} \bar{j} \bar{j}}-\epsilon^{2} \sum_{p=1}^{n} \frac{\left|h_{i j \bar{p}}\right|^{2}}{1+\epsilon h_{p \bar{p}}}
$$


We have

$$
\begin{aligned}
& b=2+\epsilon h_{1 \overline{1} 1 \overline{1}}-\epsilon^{2}\left|h_{1 \overline{1} 1}\right|^{2}\left(1+\epsilon h_{1 \overline{1}}\right)^{-1} \\
& c=1+\epsilon h_{1 \overline{1} 2 \overline{2}}-\epsilon^{2}\left|h_{2 \overline{2} 1}\right|^{2}\left(1+\epsilon h_{2 \overline{2}}\right)^{-1} \\
& d=2+\epsilon h_{2 \overline{2} 2 \overline{2}} \\
& e=1+\epsilon h_{2 \overline{2} 3 \overline{3}}
\end{aligned}
$$

Since at $q_{0}$,

$$
h_{2 \overline{2} 2 \overline{2}}=2 h_{2 \overline{2} 3 \overline{3}}=\left.2 h_{s s}\right|_{s=0}>0
$$

Therefore, $d=2 e$, and

$$
A=\left[\begin{array}{cc}
b & c x^{*} \\
c x & e B
\end{array}\right] ; \quad \text { where } \quad x^{*}=(1, \ldots, 1) \quad \text { and } B=I_{n-1}+x x^{*}
$$

Write $y^{*}=(1,0, \ldots, 0)$, and let $U$ be a unitary $(n-1)$ matrix such that $U x=\sqrt{n-1} y$. Then

$$
\left[\begin{array}{cc}
1 & 0 \\
0 & U
\end{array}\right] A\left[\begin{array}{cc}
1 & 0 \\
0 & U^{*}
\end{array}\right]=\left[\begin{array}{cc}
b & c \sqrt{n-1} y^{*} \\
c \sqrt{n-1} y & e\left(I+(n-1) y y^{*}\right)
\end{array}\right]
$$

Therefore $A$ is positive definite if and only if $b>0, e>0$, and $n b e>$ $(n-1) c^{2}$.

Since

$$
h=h(t, s)=\log \left(\frac{v-\bar{m} t^{\frac{1}{m}}}{\left(v-t^{\frac{1}{m}}\right)^{3} v^{2 m-1}} \cdot \frac{1}{(1-t-s)^{4}}\right),
$$

Let us use $h^{\prime}$ to denote $h_{t}+t h_{t t}$, and write $\eta=1-t=1-|a|^{2}$. When $t=|a|^{2} \rightarrow 0$, we have the following approximations:

$$
\begin{aligned}
h_{1 \overline{1}} & =h^{\prime}\left|w_{1}\right|^{2} \approx \eta^{2}\left[\alpha t^{\frac{1}{m}-1}+\beta t^{\frac{2}{m}-1}\right] \\
h_{2 \overline{2}} & =h_{s} \approx 6+\frac{1}{m} \\
h_{1 \overline{1} 1} & =h^{\prime} w_{11} \overline{w_{1}}+\left(h^{\prime}\right)_{t} \bar{w} w_{1}\left|w_{1}\right|^{2}=\bar{a}\left(\eta^{3}\left(h^{\prime}\right)_{t}-2 \eta^{2} h^{\prime}\right) \\
& \approx \bar{a} \eta^{3}\left[\left(\frac{1}{m}-1\right) \alpha t^{\frac{1}{m}-2}+\left(\frac{2}{m}-1\right) \beta t^{\frac{2}{m}-2}\right] \\
h_{2 \overline{2} 1} & =h_{s t} \bar{w} w_{1} \approx \bar{a} \eta m \alpha t^{\frac{1}{m}-1} \\
h_{1 \overline{1} 1 \overline{1}} & =h^{\prime}\left|w_{11}\right|^{2}+\left(h^{\prime}\right)^{\prime}\left|w_{1}\right|^{4}+\left(h^{\prime}\right)_{t}\left(w w_{11} \bar{w}^{2}+\overline{w w_{11}} w_{1}^{2}\right)
\end{aligned}
$$




$$
\begin{aligned}
& =\eta^{4}\left(h^{\prime}\right)^{\prime}-4 t \eta^{3}\left(h^{\prime}\right)_{t}+4 t \eta^{2} h^{\prime} \\
& \approx \eta^{4}\left[\left(\frac{1}{m}-1\right)^{2} \alpha t^{\frac{1}{m}-2}+\left(\frac{2}{m}-1\right)^{2} \beta t^{\frac{2}{m}-2}\right] \\
h_{1 \overline{1} 2 \overline{2}} & =\left(h_{s}\right)^{\prime}\left|w_{1}\right|^{2} \approx \eta^{2} \alpha t^{\frac{1}{m}-1} \\
h_{2 \overline{2} 3 \overline{3}} & =h_{s s} \approx 2+\frac{4}{m}-\frac{2}{m^{2}}
\end{aligned}
$$

Here $\alpha=\frac{3-\bar{m}}{m^{2}}$, and $\beta=\frac{2\left(3-\bar{m}^{2}\right)}{m^{2}}$. We have

$$
\begin{aligned}
& R_{1} \equiv h_{1 \overline{1}} h_{1 \overline{1} 1 \overline{1}}-\left|h_{1 \overline{1} 1}\right|^{2} \approx \eta^{6} t^{\frac{2}{m}-3}\left(\frac{1}{m^{2}} \alpha \beta t^{\frac{1}{m}}\right) \\
& R_{2} \equiv h_{2 \overline{2}} h_{1 \overline{1} 2 \overline{2}}-\left|h_{1 \overline{2} 2}\right|^{2} \approx\left(6+\frac{1}{m}\right) \alpha \eta^{2} t^{\frac{1}{m}-1}
\end{aligned}
$$

Therefore, $b>0, e>0$, and $c>0$. Since $0<c \leq c_{1} \equiv 1+\epsilon h_{1 \overline{1} 2 \overline{2}}$, in order to prove $n b e>(n-1) c^{2}$, it suffices to show that $Q \equiv\left(1+\epsilon h_{1 \overline{1}}\right)(n b e-(n-$ 1) $\left.c_{1}^{2}\right)>0$. Write $Q=Q_{0}+Q_{1} \epsilon+Q_{2} \epsilon^{2}+Q_{3} \epsilon^{3}$, then

$$
\begin{aligned}
& Q_{0}=n+1 \\
& Q_{1}=n h_{1 \overline{1} 1 \overline{1}}-2(n-1) h_{1 \overline{1} 2 \overline{2}}+(n+1) h_{1 \overline{1}} \approx n h_{1 \overline{1} 1 \overline{1}} \\
& Q_{2}=n R_{1}+n h_{2 \overline{2} 3 \overline{3}}\left(h_{1 \overline{1}} 2 \overline{2}+2 h_{1 \overline{1}}\right)-(n-1) h_{1 \overline{1} 2 \overline{2}}\left(h_{1 \overline{1} 2 \overline{2}}+2 h_{1 \overline{1}}\right) \approx n R_{1} \\
& Q_{3}=n h_{2 \overline{2} 3 \overline{3}} R_{1}-(n-1) h_{1 \overline{1}}\left(h_{1 \overline{1}} \overline{2}\right)^{2}
\end{aligned}
$$

Hence $Q_{1}>0, Q_{2}>0$, while

$$
Q_{3} \approx\left\{n\left(2+\frac{4}{m}-\frac{2}{m^{2}}\right) \frac{\alpha \beta}{m^{2}}-(n-1) \alpha^{3}\right\} t^{\frac{3}{m}-3}>0
$$

Therefore $A>0$. This shows that the complex curvature operator of $\omega_{\epsilon}$ is negative definite in $Y \backslash R$. For the points on $R$, the curvature tensor can be directly computed and it is also negative. Let us skip the details here. This completes the proof of the Theorem 1.

Remark. In [K-M], Kudla and Millson constructed in all dimensions compact complex ball quotient $X^{n}$ with smooth totally geodesic divisor $D$. But we do not know of any examples of a good cover $Y$ over $X$ with branching locus $D$. This amounts to looking for finite index subgroups of $\pi_{1}(X \backslash D)$ with certain property. 
Next, we consider the general case when the branching locus $D$ is not smooth but only (locally) normal crossing. In this case the complex curvature operator of $\omega_{\epsilon}$ is only quasi-negative on $Y$, since it will have some zero bisectional curvature at the singular point set $S$ of the ramification locus $R$. The computation now becomes much more complicated. We shall only give the detailed proof for the two dimensional case.

Theorem 2. Suppose that $X^{2}$ is a smooth compact quotient of the complex 2-ball, and $f: Y^{2} \rightarrow X^{2}$ is a good covering with totally geodesic branching locus $D$. Then there exists a Kähler metric on $Y$ which has negative definite complex curvature operator away from the finite set $S=\operatorname{Sing}(R)$, where $R$ is the ramification locus of $f$.

In particular, such surface $Y$ is strongly rigid.

Proof. Again the metric on $Y$ is the sum metric $\omega_{\epsilon}$ constructed in $\S 1$. It suffices to show that there exists a constant $r>0$, such that, for any $0<\epsilon<\epsilon_{0}$, the complex curvature operator of $\omega_{\epsilon}$ is negative definite in $f^{-1}\left(\cup B_{r}\left(p_{i}\right)-D\right)$, where $\left\{\ldots, p_{i}, \ldots\right\}$ is the set of nodes of $D$, and $B_{r}(p)$ is the geodesic ball of radius $r$ centered at $p$ (with respect to the canonical metric $g$ on $X$ ). Then the smooth part of $D$ can be dealt with as in Theorem 1 to give a neighborhood $V$ of $R$ such that $\omega_{\epsilon}$ is negatively curved in $V \backslash R$ for any small $\epsilon$.

Let $p_{0}$ be a node of $R$. Fix a point $p$ near $p_{0}$ which is not on $R$. Denote by $q=f(p), q_{0}=f\left(p_{0}\right)$. Let $R_{1}, R_{2}$ be the two branches of $R$ that pass through $p_{0}$, and $D_{1}, D_{2}$ be the two branches of $D$ that pass through $q_{0}$. And let $m_{i}$ be the multiplicity of $f$ along $R_{i}, i=1,2$. For simplicity, we will assume that $D_{1}$ is perpendicular to $D_{2}$ at $q_{0}$, and that $m_{1}=m_{2}$. The general case is similar, but more technical and tedious.

Again let $\left\{z_{1}, z_{2}\right\}$ be the canonical coordinate of the complex 2-ball such that $q_{0}$ is underneath the origin, and $D_{i}=\left\{z_{i}=0\right\}, i=1,2$. Choose a local inverse map $f_{1}$ of $f$ which sends $q$ to $p$, and let $\varphi$ be the local isometry on $X$ which is defined by

$$
\varphi\left(z_{1}, z_{2}\right) \equiv(x, y)=\frac{1}{1+\delta z_{1}}\left(\delta a+a z_{1}-\frac{\bar{b}}{\lambda} z_{2}, \delta b+b z_{1}+\frac{\bar{a}}{\lambda} z_{2}\right)
$$

where $q=(\delta a, \delta b)$ with $\delta>0,|a|^{2}+|b|^{2}=1$, and $\lambda=\left(1-\delta^{2}\right)^{-\frac{1}{2}}$.

$\varphi$ maps the origin to $q$. So in order to compute the curvature of $\omega_{\epsilon}$ at the point $p$, we may consider instead the metric $\left(f_{1} \circ \varphi\right)^{*} \omega_{\epsilon}=\omega_{g}+\epsilon \omega_{h}$ at 
the origin. Here

$$
\omega_{h}=\sqrt{-1} \partial \bar{\partial} h
$$

where

$$
\begin{aligned}
& h=h(t, s)=\log \left(\frac{v-\bar{m} t^{\frac{1}{m}}}{\left(v-t^{\frac{1}{m}}\right)^{3}} \frac{u-\bar{m} s^{\frac{1}{m}}}{\left(u-s^{\frac{1}{m}}\right)^{3}} \frac{1}{(u v)^{2 m-1}}\right) \\
& u=(1-s)^{\frac{1}{m}}, \quad v=(1-t)^{\frac{1}{m}}, \quad t=|x|^{2}, \quad s=|y|^{2}
\end{aligned}
$$

At the origin, we have

$$
x=\delta a, \quad y=\delta b, \quad x_{1}=\frac{a}{\lambda^{2}}, \quad x_{2}=-\frac{\bar{b}}{\lambda}, \quad y_{1}=\frac{b}{\lambda^{2}}, \quad y_{2}=\frac{\bar{a}}{\lambda}
$$

and

$$
x_{i k}=\sigma_{i k} \frac{\lambda^{2}}{|a|^{2}} \bar{x} x_{i} x_{k}, \quad y_{i k}=\sigma_{i k} \frac{\lambda^{2}}{|b|^{2}} \bar{y} y_{i} y_{k}
$$

where $\sigma_{11}=-2, \sigma_{12}=-1$, and $\sigma_{22}=0$.

Again let us use the short notation $h^{\prime}=h_{t}+t h_{t t}, h^{\prime \prime}=h_{s}+s h_{s s}$. We have

$$
\begin{gathered}
h_{i \bar{j}}=h^{\prime} x_{i} \overline{x_{j}}+h^{\prime \prime} y_{i} \overline{y_{j}}+h_{t s}\left(t_{i} s_{\bar{j}}+s_{i} t_{\bar{j}}\right) \\
h_{i \bar{j} k}=\phi_{i k} \overline{x_{j}}+\psi_{i k} \overline{y_{j}} \quad \text { with } \\
{\left[\begin{array}{c}
\phi_{i k} \\
\psi_{i k}
\end{array}\right]=S_{i k} X_{i k}=S_{i k}\left[\begin{array}{c}
x_{i} x_{k} \\
y_{i} y_{k} \\
x_{i} y_{k}+y_{i} x_{k}
\end{array}\right]}
\end{gathered}
$$

where

$$
S_{i k}=\left[\begin{array}{ccc}
\bar{x}\left(h_{t}^{\prime}+\frac{\lambda^{2} \sigma}{|a|^{2}} h^{\prime}\right) & x \bar{y}^{2}\left(h_{t s s}+\frac{\lambda^{2} \sigma}{|b|^{2}} h_{t s}\right) & \bar{y} h_{s}^{\prime} \\
\bar{x}^{2} y\left(h_{t t s}+\frac{\lambda^{2} \sigma}{|a|^{2}} h_{t s}\right) & \bar{y}\left(h_{s}^{\prime \prime}+\frac{\lambda^{2} \sigma}{|b|^{2}} h^{\prime \prime}\right) & \bar{x} h_{t}^{\prime \prime}
\end{array}\right]
$$

( $\sigma=\sigma_{i k}$ in this identity). Therefore at the origin,

$$
\sum_{p, q=1}^{2} h_{i \bar{p} k} h_{\bar{j} q \bar{l}}(I+\epsilon h)^{\bar{p} q}=\operatorname{det}(I+\epsilon h)^{-1} X_{i k}(M+\epsilon N) X_{j l}^{*}
$$

where

$$
M={ }^{t} S_{i k} \frac{1}{\lambda^{2}}\left[\begin{array}{cc}
1-t & -\bar{x} y \\
-x \bar{y} & 1-s
\end{array}\right] \overline{S_{j l}}
$$




$$
N={ }^{t} S_{i k} \frac{1}{\lambda^{6}}\left[\begin{array}{cc}
h^{\prime \prime} & -\bar{x} y h_{t s} \\
-x \bar{y} h_{t s} & h^{\prime}
\end{array}\right] \overline{S_{j l}}
$$

If we write $\sigma=\sigma_{i k}$ and $\tilde{\sigma}=\sigma_{j l}$, and use $\tilde{a}$ to denote the term obtained from $a$ with $\sigma$ in it all replaced by $\tilde{\sigma}$. Also denote the entries of $S$ by

$$
\begin{aligned}
& S=S_{i k}=\left[\begin{array}{ccc}
\bar{x} a_{1} & x \bar{y}^{2} b_{2} & \bar{y} h_{s}^{\prime} \\
\bar{x}^{2} y b_{1} & \bar{y} a_{2} & \bar{x} h_{t}^{\prime \prime}
\end{array}\right] \\
& \widetilde{S}=S_{j l}=\left[\begin{array}{ccc}
\bar{x} \widetilde{a_{1}} & x \bar{y}^{2} \widetilde{b_{2}} & \bar{y} h_{s}^{\prime} \\
\bar{x} \tilde{y} \tilde{b_{1}} & \bar{y} \widetilde{a_{2}} & \bar{x} h_{t}^{\prime \prime}
\end{array}\right]
\end{aligned}
$$

Then we have

$$
\begin{aligned}
& M=\frac{1}{\lambda^{2}}\left[\begin{array}{lll}
c_{1} & d_{1} & d_{2} \\
\frac{\widetilde{d_{1}}}{\widetilde{d_{2}}} & \frac{c_{2}}{\widetilde{d_{3}}} & d_{3}
\end{array}\right] \quad \text { where } \\
& c_{1}=t(1-t) a_{1} \tilde{a_{1}}+t^{2} s(1-s) b_{1} \tilde{b_{1}}-t^{2} s\left(a_{1} \tilde{b_{1}}+b_{1} \tilde{a_{1}}\right) \\
& c_{2}=t s^{2}(1-t) b_{2} \tilde{b_{2}}+s(1-s) a_{2} \tilde{a_{2}}-t s^{2}\left(a_{2} \tilde{b_{2}}+b_{2} \tilde{a_{2}}\right) \\
& c_{3}=s(1-t)\left(h_{s}^{\prime}\right)^{2}+t(1-s)\left(h_{t}^{\prime \prime}\right)^{2}-2 t s h_{s}^{\prime} h_{t}^{\prime \prime} \\
& d_{1}=\bar{x}^{2} y^{2}\left[(1-t) a_{1} \tilde{b_{2}}+(1-s) b_{1} \tilde{a_{2}}-\left(a_{1} \tilde{a_{2}}+t s b_{1} \tilde{b_{2}}\right)\right] \\
& d_{2}=\bar{x} y\left[(1-t) a_{1} h_{s}^{\prime}+t(1-s) b_{1} h_{t}^{\prime \prime}-t\left(a_{1} h_{t}^{\prime \prime}+s b_{1} h_{s}^{\prime}\right)\right] \\
& d_{3}=x \bar{y}\left[s(1-t) b_{2} h_{s}^{\prime}+(1-s) a_{2} h_{t}^{\prime \prime}-s\left(a_{2} h_{s}^{\prime}+t b_{2} h_{t}^{\prime \prime}\right)\right] \\
& N=\frac{1}{\lambda^{6}}\left[\begin{array}{lll}
c_{1}^{\prime} & d_{1}^{\prime} & d_{2}^{\prime} \\
\widetilde{\widetilde{d_{1}^{\prime}}} & \frac{c_{2}^{\prime}}{\widetilde{d_{2}^{\prime}}} & d_{3}^{\prime} \\
\widetilde{d_{3}^{\prime}} & c_{3}^{\prime}
\end{array}\right] \quad \text { where } \\
& c_{1}^{\prime}=t h^{\prime \prime} a_{1} \tilde{a_{1}}+t^{2} s^{\prime} b_{1} \tilde{b_{1}}-t^{2} s h_{t s}\left(a_{1} \tilde{b_{1}}+b_{1} \tilde{a_{1}}\right) \\
& c_{2}^{\prime}=t s^{2} h^{\prime \prime} b_{2} \tilde{b_{2}}+s h^{\prime} a_{2} \tilde{a_{2}}-t s^{2} h_{t s}\left(a_{2} \tilde{b_{2}}+b_{2} \tilde{a_{2}}\right) \\
& c_{3}^{\prime}=s h^{\prime \prime}\left(h_{s}^{\prime}\right)^{2}+t h^{\prime}\left(h_{t}^{\prime \prime}\right)^{2}-2 t s h_{t s} h_{s}^{\prime} h_{t}^{\prime \prime} \\
& d_{1}^{\prime}=\bar{x}^{2} y^{2}\left[h^{\prime \prime} a_{1} \tilde{b_{2}}+h^{\prime} b_{1} \tilde{a_{2}}-h_{t s}\left(a_{1} \tilde{a_{2}}+t s b_{1} \tilde{b_{2}}\right)\right] \\
& d_{2}^{\prime}=\bar{x} y\left[h^{\prime \prime} a_{1} h_{s}^{\prime}+t h^{\prime} b_{1} h_{t}^{\prime \prime}-t h_{t s}\left(a_{1} h_{t}^{\prime \prime}+s b_{1} h_{s}^{\prime}\right)\right] \\
& d_{3}^{\prime}=x \bar{y}\left[s h^{\prime \prime} b_{2} h_{s}^{\prime}+h^{\prime} a_{2} h_{t}^{\prime \prime}-s h_{t s}\left(a_{2} h_{s}^{\prime}+t b_{2} h_{t}^{\prime \prime}\right)\right]
\end{aligned}
$$

Next, we compute at the origin:

$$
h_{i \bar{j} k \bar{l}}={ }^{t} X_{i k} H \overline{X_{j l}}
$$


where

$$
\begin{aligned}
H=\left[\begin{array}{lll}
\frac{e_{1}}{\widetilde{\widetilde{f}_{1}}} & f_{1} & f_{2} \\
\frac{e_{2}}{\widetilde{f_{2}}} & f_{3} \\
\widetilde{f_{3}} & e_{3}
\end{array}\right] \quad \text { with } \\
e_{1}=\left(h^{\prime}\right)^{\prime}+(\sigma+\tilde{\sigma}) \lambda^{2}|a|^{-2} t h_{t}^{\prime}+\sigma \tilde{\sigma} \lambda^{4}|a|^{-4} t h^{\prime} \\
e_{2}=\left(h^{\prime \prime}\right)^{\prime \prime}+(\sigma+\tilde{\sigma}) \lambda^{2}|b|^{-2} s h_{s}^{\prime \prime}+\sigma \tilde{\sigma} \lambda^{4}|b|^{-4} s h^{\prime \prime} \\
e_{3}=\left(h^{\prime}\right)^{\prime \prime} \\
f_{1}=\bar{x}^{2} y^{2}\left[h_{t t s s}+\sigma \lambda^{2}|a|^{-2} h_{t s s}+\tilde{\sigma} \lambda^{2}|b|^{-2} h_{t t s}+\sigma \tilde{\sigma} \lambda^{4}|a b|^{-2} h_{t s}\right] \\
f_{2}=\bar{x} y\left[h_{t s}^{\prime}+\sigma \lambda^{2}|a|^{-2} h_{s}^{\prime}\right] \\
f_{3}=x \bar{y}\left[h_{t s}^{\prime \prime}+\sigma \lambda^{2}|b|^{-2} h_{t}^{\prime \prime}\right]
\end{aligned}
$$

Since at the origin, the canonical metric $g$ is normal for the coordinate $\left\{z_{1}, z_{2}\right\}$, so the curvature components of the metric $g+\epsilon h$ at the origin is

$$
\begin{aligned}
-R_{i \bar{j} k \bar{l}} & =g_{i \bar{j} k \bar{l}}+\epsilon h_{i \bar{j} k \bar{l}}-\epsilon^{2} \sum_{p, q=1}^{2} h_{i \bar{p} k} h_{\bar{j} q \bar{l}}(I+\epsilon h)^{\bar{p} q} \\
& =-R(g)_{\bar{i} \bar{j} k \bar{l}}+{ }^{t} X_{i k}\left(\epsilon H-\epsilon^{2} \operatorname{det}(I+\epsilon h)^{-1}(M+\epsilon N)\right) \overline{X_{j l}}
\end{aligned}
$$

Write $e_{i}=\frac{\partial}{\partial z_{i}}$, and let

$$
u_{1}=-y_{2} e_{1}+y_{1} e_{2}, \quad u_{2}=x_{2} e_{1}-x_{1} e_{2}, \gamma=x_{1} y_{2}-x_{2} y_{1}
$$

Then $e_{i}=-\gamma^{-1}\left(x_{i} u_{1}+y_{i} u_{2}\right)$. Hence

$$
\begin{gathered}
-R(g)_{i \bar{j} k \bar{l}}={ }^{t} X_{i k} G \overline{X_{j l}} \\
G=\gamma^{-4}\left[\begin{array}{ccc}
2\left|u_{1}\right|^{4} & 2\left(u_{1}, \overline{u_{2}}\right)^{2} & 2\left|u_{1}\right|^{2}\left(u_{1}, \overline{u_{2}}\right) \\
2\left(u_{2}, \overline{u_{1}}\right)^{2} & 2\left|u_{2}\right|^{4} & 2\left|u_{2}\right|^{2}\left(u_{2}, \overline{u_{1}}\right) \\
2\left|u_{1}\right|^{2}\left(u_{2}, \overline{u_{1}}\right) & 2\left|u_{2}\right|^{2}\left(u_{1}, \overline{u_{2}}\right) & \left|u_{1}\right|^{2}\left|u_{2}\right|^{2}+\left|\left(u_{1}, \overline{u_{2}}\right)\right|^{2}
\end{array}\right]
\end{gathered}
$$

Plug in the values of $x_{i}, y_{j}$ at the origin, we get

$$
G=\lambda^{8}\left[\begin{array}{ccc}
2(1-s)^{2} & 2 \bar{x}^{2} y^{2} & 2(1-s) \bar{x} y \\
2 x^{2} \bar{y}^{2} & 2(1-t)^{2} & 2(1-t) x \bar{y} \\
2(1-s) x \bar{y} & 2(1-t) \bar{x} y & 1-t-s+2 t s
\end{array}\right]
$$

Therefore

$$
-R_{i \bar{j} k \bar{l}}=\operatorname{det}(I+\epsilon h)^{-1} \cdot{ }^{t} X_{i k} P \overline{X_{j l}}
$$




$$
\begin{aligned}
P & =\operatorname{det}(I+\epsilon h) \cdot(G+\epsilon H)-\epsilon^{2}(M+\epsilon N) \\
& =P_{0}+P_{1} \epsilon+P_{2} \epsilon^{2}+P_{3} \epsilon^{3}, \quad \text { where } \\
P_{0} & =G \\
P_{1} & =\operatorname{tr}(h) G+H \\
P_{2} & =\operatorname{det}(h) G+\operatorname{tr}(h) H-M \\
P_{3} & =\operatorname{det}(h) H-N
\end{aligned}
$$

Write

$$
P=P^{(1)}+\sigma P^{(2)}+\tilde{\sigma} P^{(2) *}+\sigma \tilde{\sigma} P^{(3)}
$$

where $P^{(1)}$ and $P^{(3)}$ are hermitian symmetric, and define

$$
\hat{P}=P^{(1)}+U P^{(2)}+P^{(2) *} U^{*}+U P^{(3)} U^{*}
$$

where

$$
U=-\left[\begin{array}{ccc}
2|a|^{2} & 0 & 2 \bar{a} b \\
0 & 2|b|^{2} & 2 a \bar{b} \\
a \bar{b} & \bar{a} b & 1
\end{array}\right]
$$

If we write

$$
\hat{P}=\left[\begin{array}{lll}
A & D & E \\
\bar{D} & C & F \\
\bar{E} & \bar{F} & B
\end{array}\right]
$$

then it is not hard to verify that up to a common positive scalar factor, the entries are just the component of the curvature tensor under the tangent frame $\left\{u_{1}, u_{2}\right\}$. On the other hand, the complex curvature operator $-Q$ is a hermitian bilinear form over the tensor of the tangent space $T_{q_{0}} \otimes \overline{T_{q_{0}}}$. Under the basis $u_{i} \otimes \overline{u_{j}}, Q$ becomes

$$
Q=\left[\begin{array}{llll}
A & B & \bar{E} & E \\
B & C & F & \bar{F} \\
E & \bar{F} & B & D \\
\bar{E} & F & \bar{D} & B
\end{array}\right]
$$

Since $Q=Q_{0}+Q_{1} \epsilon+Q_{2} \epsilon^{2}+Q_{3} \epsilon^{3}$, and $-Q_{0}$, the curvature operator of $g$, is negative definite, therefore, it suffices to show that $Q_{i}>0$ for $1 \leq i \leq 3$ when the point $p$ is very close to the node $p_{0}$, or, equivalently, when $\delta$ is sufficiently small. We want to write down the leading terms of the entries of $\hat{P}$ when $\delta \rightarrow 0$. Without loss of generality, we may restrict ourselves to the following two cases:

Case I: $\delta \rightarrow 0, a \rightarrow a_{0}, b \rightarrow b_{0}, a_{0} b_{0} \neq 0$. 
Case II: $\delta \rightarrow 0, a \rightarrow 0$ (hence $|b| \rightarrow 1$ ).

In the first case, $t$ and $s$ approach zero at the same speed, while in the second case, $t \ll s$.

We shall need the following simple fact from linear algebra, the proof is omitted.

Lemma. If the entries of $\hat{P}$ satisfy

$$
\begin{aligned}
A, B, C>0 ; \quad(1-r)^{2} A C>B^{2} ; \quad & (1-r) B>|D| ; \\
& r^{2} A B>4|E|^{2} ; \quad r^{2} B C>4|F|^{2}
\end{aligned}
$$

for some constant $0<r<1$. Then $Q>0$.

In the following, we shall compute the leading terms in the asymptotic expansions of the entries of $\hat{P}$ and check that they satisfy the conditions in the above lemma.

Case I: $t \sim s$.

We shall use $\approx$ to denote the first two leading terms approximation, where the second term is equivalent to the first one multiplied by $\delta^{\frac{2}{m}}$. We have

$$
h=h(t, s)=\log \left(\frac{v-\bar{m} t^{\frac{1}{m}}}{v-t^{\frac{1}{m}}} \cdot \frac{u-\bar{m} s^{\frac{1}{m}}}{u-s^{\frac{1}{m}}} \cdot(u v)^{1-2 m}\right)
$$

where $u=(1-t)^{\frac{1}{m}}, v=(1-s)^{\frac{1}{m}}$. Again let $\alpha=\frac{3-\bar{m}}{m^{2}}, \beta=\frac{2\left(3-\bar{m}^{2}\right)}{m^{2}}$. Also let $\alpha_{1}=\left(\frac{1}{m}-1\right) \alpha, \alpha_{2}=\left(\frac{1}{m}-1\right)^{2} \alpha$, and $\beta_{1}=\left(\frac{2}{m}-1\right) \beta, \beta_{2}=\left(\frac{2}{m}-1\right)^{2} \beta$. We compute

$$
\begin{aligned}
h^{\prime} & \approx \alpha t^{\frac{1}{m}-1}+\beta t^{\frac{2}{m}-1} \\
h^{\prime \prime} & \approx \alpha s^{\frac{1}{m}-1}+\beta s^{\frac{2}{m}-1} \\
h_{t s} & \approx h^{\prime}+h^{\prime \prime} \\
\left(h^{\prime}\right)_{t} & \approx h_{t t s} \approx \alpha_{1} t^{\frac{1}{m}-2}+\beta_{1} t^{\frac{2}{m}-1} \\
\left(h^{\prime}\right)^{\prime} & \approx \alpha_{2} t^{\frac{1}{m}-2}+\beta_{2} t^{\frac{2}{m}-1} \\
\left(h^{\prime}\right)_{t s} & \approx \frac{1}{m} \alpha_{1} t^{\frac{1}{m}-2}+\frac{2}{m} \beta_{1} t^{\frac{2}{m}-2} \\
\left(h^{\prime}\right)_{s} & \approx \frac{1}{m} \alpha_{1} t^{\frac{1}{m}-1}+\frac{2}{m} \beta_{1} t^{\frac{2}{m}-1}+\alpha s^{\frac{1}{m}-1}+\beta s^{\frac{2}{m}-1}
\end{aligned}
$$




$$
\begin{aligned}
\left(h^{\prime}\right)^{\prime \prime} & \approx \frac{1}{m} \alpha\left(t^{\frac{1}{m}-1}+s^{\frac{1}{m}-1}\right)+\frac{2}{m} \beta\left(t^{\frac{2}{m}-1}+s^{\frac{2}{m}-1}\right) \\
h_{t t s s} & \approx\left(\frac{1}{m}+1\right) \alpha_{1}\left(t^{\frac{1}{m}-2}+s^{\frac{1}{m}-2}\right)+\left(\frac{2}{m}+1\right) \beta_{1}\left(t^{\frac{2}{m}-1}+s^{\frac{2}{m}-1}\right)
\end{aligned}
$$

Since $\lambda \approx 1$, we also have

$$
\begin{aligned}
\operatorname{tr}(h) & =\frac{1}{\lambda^{2}}\left((1-t) h^{\prime}+(1-s) h^{\prime \prime}-2 s t h_{t s}\right) \approx h^{\prime}+h^{\prime \prime} \\
\operatorname{det}(h) & =\frac{1}{\lambda^{6}}\left(h^{\prime} h^{\prime \prime}-s t h_{t s}^{2}\right) \approx h^{\prime} h^{\prime \prime} \\
a_{1} & \approx h_{t}^{\prime}+\sigma \frac{h^{\prime}}{|a|^{2}}, \quad b_{1} \approx h_{t}^{\prime}+\sigma \frac{h_{t s}}{|a|^{2}} \\
a_{2} & \approx h_{s}^{\prime \prime}+\sigma \frac{h^{\prime \prime}}{|b|^{2}}, \quad b_{2} \approx h_{s}^{\prime \prime}+\sigma \frac{h_{t s}}{|b|^{2}}
\end{aligned}
$$

Therefore

$$
\begin{aligned}
& M^{(1)} \approx {\left[\begin{array}{ccc}
t\left(h_{t}^{\prime}\right)^{2} & \bar{x}^{2} y^{2} h_{t}^{\prime} h_{s}^{\prime \prime} & \bar{x} y h_{s}^{\prime} h_{t}^{\prime} \\
* & s\left(h_{s}^{\prime \prime}\right)^{2} & x \bar{y} h_{t}^{\prime \prime} h_{s}^{\prime \prime} \\
* & * & s\left(h_{s}^{\prime}\right)^{2}+t\left(h_{t}^{\prime \prime}\right)^{2}
\end{array}\right] } \\
& M^{(2)} \approx\left[\begin{array}{ccc}
\frac{t}{|a|^{2}} h^{\prime} h_{t}^{\prime} & \frac{\bar{x}^{2} y^{2}}{|a|^{2}} h_{t s} h_{s}^{\prime \prime} & \frac{\bar{x} y}{|a|^{2}} h^{\prime} h_{s}^{\prime} \\
\frac{x^{2} \bar{y}^{2}}{|b|^{2}} h_{t s} h_{t}^{\prime} & \frac{s}{|b|^{2}} h^{\prime \prime} h_{s}^{\prime \prime} & \frac{x \bar{y}}{|b|^{2}} h^{\prime \prime} h_{t}^{\prime \prime} \\
0 & 0 & 0
\end{array}\right] \\
& M^{(3)} \approx\left[\begin{array}{ccc}
\frac{t}{|a|^{4}}\left(h^{\prime}\right)^{2} & \frac{\bar{x}^{2} y^{2}}{|a|^{2}|b|^{2}}\left(h_{t s}^{2}-h^{\prime} h^{\prime \prime}\right) & 0 \\
* & \frac{s}{|b|^{4}}\left(h^{\prime \prime}\right)^{2} & 0 \\
0 & 0 & 0
\end{array}\right]
\end{aligned}
$$

We have

$$
\begin{gathered}
U M^{(2)} \approx-\left[\begin{array}{ccc}
2 t h^{\prime} h_{t}^{\prime} & 2 \bar{x}^{2} y^{2} h_{t s} h_{s}^{\prime \prime} & 2 \bar{x} y h^{\prime} h_{s}^{\prime} \\
2 x^{2} \bar{y}^{2} h_{t s} h_{t}^{\prime} & 2 s h^{\prime \prime} h_{s}^{\prime \prime} & 2 x \bar{y} h^{\prime \prime} h_{t}^{\prime \prime} \\
x \bar{y} h^{\prime} h_{t}^{\prime} & \bar{x} y h^{\prime \prime} h_{s}^{\prime \prime} & s h^{\prime} h_{s}^{\prime}+t h^{\prime \prime} h_{t}^{\prime \prime}
\end{array}\right] \\
U M^{(3)} U^{*} \approx\left[\begin{array}{ccc}
4 t\left(h^{\prime}\right)^{2} & 4 \bar{x}^{2} y^{2}\left(h_{t s}^{2}-h^{\prime} h^{\prime \prime}\right) & 2 \bar{x} y\left(h^{\prime}\right)^{2} \\
* & 4 s\left(h^{\prime \prime}\right)^{2} & 2 x \bar{y}\left(h^{\prime \prime}\right)^{2} \\
* & * & s\left(h^{\prime}\right)^{2}+t\left(h^{\prime \prime}\right)^{2}
\end{array}\right]
\end{gathered}
$$


Therefore, by comparing the orders, we know that $\hat{M}$ is

$$
\hat{M} \approx\left[\begin{array}{ccc}
t\left(h_{t}^{\prime}\right)^{2} & \bar{x}^{2} y^{2} h_{t}^{\prime} h_{s}^{\prime \prime} & \bar{x} y\left(h_{s}^{\prime}-h^{\prime}\right) h_{t}^{\prime} \\
* & s\left(h_{s}^{\prime \prime}\right)^{2} & x \bar{y}\left(h_{t}^{\prime \prime}-h^{\prime \prime}\right) h_{s}^{\prime \prime} \\
* & * & s\left(h_{s}^{\prime}-h^{\prime}\right)^{2}+t\left(h_{t}^{\prime \prime}-h^{\prime \prime}\right)^{2}
\end{array}\right]
$$

Similarly,

$$
\begin{gathered}
N^{(1)} \approx\left[\begin{array}{ccc}
t h^{\prime \prime}\left(h_{t}^{\prime}\right)^{2} & \bar{x}^{2} y^{2}\left(h^{\prime}+h^{\prime \prime}-h_{t s}\right) h_{t}^{\prime} h_{s}^{\prime \prime} & \bar{x} y h^{\prime \prime} h_{s}^{\prime} h_{t}^{\prime} \\
* & s h^{\prime}\left(h_{s}^{\prime \prime}\right)^{2} & x \bar{y} h^{\prime} h_{t}^{\prime \prime} h_{s}^{\prime \prime} \\
* & * & s h^{\prime \prime}\left(h_{s}^{\prime}\right)^{2}+t h^{\prime}\left(h_{t}^{\prime \prime}\right)^{2}
\end{array}\right] \\
N^{(2)} \approx\left[\begin{array}{ccc}
\frac{t}{|a|^{2}} h^{\prime} h^{\prime \prime} h_{t}^{\prime} & \frac{\bar{x}^{2} y^{2}}{|a|^{2}} h^{\prime} h^{\prime \prime} h_{s}^{\prime \prime} & \frac{\bar{x} y}{|a|^{2}} h^{\prime} h^{\prime \prime} h_{s}^{\prime} \\
\frac{x^{2} \bar{y}^{2}}{|b|^{2}} h^{\prime} h^{\prime \prime} h_{t}^{\prime} & \frac{s}{|b|^{2}} h^{\prime} h^{\prime \prime} h_{s}^{\prime \prime} & \frac{x \bar{y}}{|b|^{2}} h^{\prime} h^{\prime \prime} h_{t}^{\prime \prime} \\
0 & 0 & 0
\end{array}\right] \\
N^{(3)} \approx\left[\begin{array}{ccc}
\frac{t}{|a|^{4}}\left(h^{\prime}\right)^{2} h^{\prime \prime} & \frac{\bar{x}^{2} y^{2}}{|a|^{2}|b|^{2}} h^{\prime} h^{\prime \prime} h_{t s} & 0 \\
* & \frac{s}{|b|^{4}} h^{\prime}\left(h^{\prime \prime}\right)^{2} & 0 \\
0 & 0 & 0
\end{array}\right]
\end{gathered}
$$

Hence

$$
\begin{gathered}
U N^{(2)} \approx-\left[\begin{array}{ccc}
2 t h^{\prime} h^{\prime \prime} h_{t}^{\prime} & 2 \bar{x}^{2} y^{2} h^{\prime} h^{\prime \prime} h_{s}^{\prime \prime} & 2 \bar{x} y h^{\prime} h^{\prime \prime} h_{s}^{\prime} \\
2 x^{2} \bar{y}^{2} h^{\prime} h^{\prime \prime} h_{t}^{\prime} & 2 s h^{\prime} h^{\prime \prime} h_{s}^{\prime \prime} & 2 x \bar{y} h^{\prime} h^{\prime \prime} h_{t}^{\prime \prime} \\
x \bar{y} h^{\prime} h^{\prime \prime} h_{t}^{\prime} & \bar{x} y h^{\prime} h^{\prime \prime} h_{s}^{\prime \prime} & h^{\prime} h^{\prime \prime}\left(s h_{s}^{\prime}+t h_{t}^{\prime \prime}\right)
\end{array}\right] \\
U N^{(3)} U^{*} \approx\left[\begin{array}{ccc}
4 t\left(h^{\prime}\right)^{2} h^{\prime \prime} & 4 \bar{x}^{2} y^{2} h^{\prime} h^{\prime \prime} h_{t s} & 2 \bar{x} y\left(h^{\prime}\right)^{2} h^{\prime \prime} \\
* & 4 s h^{\prime}\left(h^{\prime \prime}\right)^{2} & 2 x \bar{y} h^{\prime}\left(h^{\prime \prime}\right)^{2} \\
* & * & h^{\prime} h^{\prime \prime}\left(s h^{\prime}+t h^{\prime \prime}\right)
\end{array}\right]
\end{gathered}
$$

So $\hat{N}$ becomes

$$
\hat{N} \approx\left[\begin{array}{ccc}
t h^{\prime \prime}\left(h_{t}^{\prime}\right)^{2} & \bar{x}^{2} y^{2}\left(h^{\prime}+h^{\prime \prime}-h_{t s}\right) h_{t}^{\prime} h_{s}^{\prime \prime} & \bar{x} y h^{\prime} h_{t}^{\prime}\left(h_{s}^{\prime}-h^{\prime}\right) \\
* & s h^{\prime}\left(h_{s}^{\prime \prime}\right)^{2} & x \bar{y} h^{\prime} h_{s}^{\prime \prime}\left(h_{t}^{\prime \prime}-h^{\prime \prime}\right) \\
* & * & s h^{\prime \prime}\left(h_{s}^{\prime}-h^{\prime}\right)^{2}+t h^{\prime}\left(h_{t}^{\prime \prime}-h^{\prime \prime}\right)^{2}
\end{array}\right]
$$

We also have

$$
H^{(1)}=\left[\begin{array}{ccc}
\left(h^{\prime}\right)^{\prime} & \bar{x}^{2} y^{2} h_{t t s s} & \bar{x} y h_{t s}^{\prime} \\
* & \left(h^{\prime \prime}\right)^{\prime \prime} & x \bar{y} h_{t s}^{\prime \prime} \\
* & * & \left(h^{\prime}\right)^{\prime \prime}
\end{array}\right]
$$




$$
\begin{gathered}
H^{(2)}=\lambda^{2}\left[\begin{array}{ccc}
\frac{t}{|a|^{2}} h_{t}^{\prime} & \frac{\bar{x}^{2} y^{2}}{|a|^{2}} h_{t s s} & \frac{\bar{x} y}{|a|^{2}} h_{s}^{\prime} \\
\frac{x^{2} \bar{y}^{2}}{|b|^{2}} h_{t t s} & \frac{s}{|b|^{2}} h_{s}^{\prime \prime} & \frac{x \bar{y}}{|b|^{2}} h_{t}^{\prime \prime} \\
0 & 0 & 0
\end{array}\right] \\
H^{(3)}=\lambda^{4}\left[\begin{array}{ccc}
\frac{t}{|a|^{4}} h^{\prime} & \frac{\bar{x}^{2} y^{2}}{|a|^{2}|b|^{2}} h_{t s} & 0 \\
* & \frac{s}{|b|^{4}} h^{\prime \prime} & 0 \\
0 & 0 & 0
\end{array}\right]
\end{gathered}
$$

Hence

$$
\begin{gathered}
U H^{(2)} \approx-\left[\begin{array}{ccc}
2 t h_{t}^{\prime} & 2 \bar{x}^{2} y^{2} h_{t s s} & 2 \bar{x} y h_{s}^{\prime} \\
2 x^{2} \bar{y}^{2} h_{t t s} & 2 s h_{s}^{\prime \prime} & 2 x \bar{y} h_{t}^{\prime \prime} \\
x \bar{y} h_{t}^{\prime} & \bar{x} y h_{s}^{\prime \prime} & s h_{s}^{\prime}+t h_{t}^{\prime \prime}
\end{array}\right] \\
U H^{(3)} U^{*} \approx\left[\begin{array}{ccc}
4 t h^{\prime} & 4 \bar{x}^{2} y^{2} h_{t s} & 2 \bar{x} y h^{\prime} \\
* & 4 s h^{\prime \prime} & 2 x \bar{y} h^{\prime \prime} \\
* & * & s h^{\prime}+t h^{\prime \prime}
\end{array}\right]
\end{gathered}
$$

Therefore $\hat{H}$ is

$$
\hat{H}=\left[\begin{array}{ccc}
\left(h^{\prime}\right)^{\prime} & \bar{x}^{2} y^{2}\left(h_{t t s s}-2 h_{t s s}-2 h_{t t s}\right) & \bar{x} y\left(h_{t s}^{\prime}-h_{t}^{\prime}\right) \\
* & \left(h^{\prime \prime}\right)^{\prime \prime} & x \bar{y}\left(h_{t s}^{\prime \prime}-h_{s}^{\prime \prime}\right) \\
* & * & \left(h^{\prime}\right)^{\prime \prime}
\end{array}\right]
$$

While

$$
G \approx\left[\begin{array}{ccc}
2 & 2 \bar{x}^{2} y^{2} & 2 \bar{x} y \\
* & 2 & 2 x \bar{y} \\
* & * & 1
\end{array}\right]
$$

Note that in all cases, the order of the entries of $X^{\prime}$ is no greater than the order of the corresponding entries in $X^{(1)}$, for $X=M, N$, or $H$.

For $\hat{H}$, the entries satisfy

$$
A \sim C \sim t^{\frac{1}{m}-2} ; \quad B \sim t^{\frac{1}{m}-1} ; \quad|D| \leq t^{\frac{1}{m}} ; \quad|E|,|F| \leq t^{\frac{1}{m}-1}
$$

Here we omit the positive constant coefficients. So we have $A>0, C>0$, $B>0$, and $A C \gg B^{2} \gg|D|^{2}, A B \gg|E|^{2}, \quad B C \gg|F|^{2}$, hence the associated $Q_{\hat{H}}>0$, and $Q_{1}=\operatorname{tr}(h) Q_{G}+Q_{\hat{H}}>0$.

For $\widehat{P_{2}}=\operatorname{det}(h) G+\operatorname{tr}(h) \hat{H}-\hat{M}$, we have

$$
\widehat{P_{2}} \approx\left[\begin{array}{ccc}
h^{\prime \prime}\left(h^{\prime}\right)^{\prime} & -\bar{x}^{2} y^{2} h_{t}^{\prime} h_{s}^{\prime \prime} & \bar{x} y\left[h_{t s}\left(h_{t s}^{\prime}-h_{t}^{\prime}\right)+h_{t}^{\prime}\left(h^{\prime}-h_{s}^{\prime}\right)\right] \\
* & h^{\prime}\left(h^{\prime \prime}\right)^{\prime \prime} & x \bar{y}\left[h_{t s}\left(h_{t s}^{\prime \prime}-h_{s}^{\prime \prime}\right)+h_{s}^{\prime \prime}\left(h^{\prime \prime}-h_{t}^{\prime \prime}\right)\right] \\
* & * & h^{\prime} h^{\prime \prime}+h_{t s}\left(h^{\prime}\right)^{\prime \prime}
\end{array}\right]
$$


So the entries satisfy

$$
A \sim C \sim t^{\frac{2}{m}-3} ; B \sim t^{\frac{2}{m}-2} ;|D|,|E|,|F| \leq t^{\frac{2}{m_{-}}-2}
$$

Again we omit the positive constant coefficients here. Therefore $A>0$, $C>0, B>0$, and $A C \gg B^{2}, A B \gg|E|^{2}, C B \gg|F|^{2}$. Also

$$
\begin{gathered}
|D| \approx t s\left|h_{t}^{\prime} h_{s}^{\prime \prime}\right| \approx \alpha_{1}^{2}(t s)^{\frac{1}{m}-1} \\
B>h^{\prime} h^{\prime \prime} \approx \alpha^{2}(t s)^{\frac{1}{m}-1}
\end{gathered}
$$

Since $\alpha_{1}=\left(\frac{1}{m}-1\right) \alpha, r B>|D|$ for some constant $0<r<1$. Therefore, $Q_{2}>0$.

For $\widehat{P_{3}}=\operatorname{det}(h) \hat{H}-\hat{N}$, since $h^{\prime}+h^{\prime \prime}-h_{t s}=O\left(t^{\frac{3}{m}-1}\right)$, we have

$$
\widehat{P_{3}} \approx
$$

$$
\left[\begin{array}{ccc}
h^{\prime \prime}\left[h^{\prime}\left(h^{\prime}\right)^{\prime}-t\left(h_{t}^{\prime}\right)^{2}\right] & -\bar{x}^{2} y^{2}\left(h^{\prime}+h^{\prime \prime}-h_{t s}\right) h_{t}^{\prime} h_{s}^{\prime \prime} & \bar{x} y h^{\prime \prime}\left(h^{\prime} h_{t s}^{\prime}-h_{t}^{\prime} h_{s}^{\prime}\right) \\
* & h^{\prime}\left[h^{\prime \prime}\left(h^{\prime \prime}\right)^{\prime \prime}-s\left(h_{s}^{\prime \prime}\right)^{2}\right] & x \bar{y} h^{\prime}\left(h^{\prime \prime} h_{t s}^{\prime \prime}-h_{t}^{\prime \prime} h_{s}^{\prime \prime}\right) \\
* & * & h^{\prime} h^{\prime \prime}\left(h^{\prime}\right)^{\prime \prime}
\end{array}\right]
$$

Since

$$
h^{\prime}\left(h^{\prime}\right)^{\prime}-t\left(h_{t}^{\prime}\right)^{2} \sim\left(\alpha \beta_{2}+\alpha_{2} \beta-2 \alpha_{1} \beta_{1}\right) t^{\frac{3}{m}-3} \sim \frac{\alpha \beta}{m^{2}} t^{\frac{3}{m}-3}>0
$$

Therefore, $A>0, C>0, B>0$, and

$$
A \sim C \sim t^{\frac{4}{m}-4}, \quad B \sim|E| \sim|F| \sim t^{\frac{3}{m}-3},|D| \sim t^{\frac{5}{m}-3}
$$

So they satisfy the conditions of the Lemma. Hence $Q_{3}>0$. This completes the proof of the first case.

Case II: $t \ll s$.

In this case, $t \rightarrow 0, s \rightarrow 0$, and $\frac{t}{s} \rightarrow 0$. In the following, we shall use $\sim$ (or $\approx$ ) to denote the approximation of the ( first two) leading terms. We have

$$
\begin{aligned}
h^{\prime} & \approx \frac{\alpha}{v} t^{\frac{1}{m}-1}+\beta t^{\frac{2}{m}-1} \\
h^{\prime \prime} & \approx \alpha s^{\frac{1}{m}-1}+\beta s^{\frac{2}{m}-1} \\
h_{t s} & \approx \frac{\alpha}{v^{m+1}} t^{\frac{1}{m}-1}+\beta t^{\frac{2}{m}-1}+\alpha s^{\frac{1}{m}-1}
\end{aligned}
$$




$$
\begin{aligned}
\left(h^{\prime}\right)_{s} & \approx \frac{\alpha}{m v^{m+1}} t^{\frac{1}{m}-1}+\frac{2 \beta}{m} t^{\frac{2}{m}-1}+\alpha s^{\frac{1}{m}-1} \\
\left(h^{\prime \prime}\right)_{t} & \approx \frac{\alpha}{v^{2 m+1}} t^{\frac{1}{m}-1}+\beta t^{\frac{2}{m}-1}+\frac{\alpha}{m} s^{\frac{1}{m}-1} \\
\left(h^{\prime}\right)_{t} & \approx v^{m} h_{t t s} \approx \frac{\alpha_{1}}{v} t^{\frac{1}{m}-2}+\beta_{1} t^{\frac{2}{m}-2} \\
\left(h^{\prime \prime}\right)_{s} & \approx \alpha_{1} s^{\frac{1}{m}-2}+\beta_{1} s^{\frac{2}{m}-2} \\
h_{t s s} & \approx\left(\alpha_{1} s^{\frac{1}{m}-2}+\frac{\left(1+\frac{1}{m}\right) \alpha}{v^{2 m+1}} t^{\frac{1}{m}-1}\right)+\beta_{1} s^{\frac{2}{m}-2}+\left(1+\frac{2}{m}\right) \alpha t^{\frac{2}{m}-1} \\
\left(h^{\prime}\right)_{t s} & \approx \frac{\alpha_{1}}{m v^{m+1}} t^{\frac{1}{m}-2}+\frac{2 \beta_{1}}{m} t^{\frac{2}{m}-2} \\
\left(h^{\prime \prime}\right)_{t s} & \sim\left(\frac{\alpha_{1}}{m} s^{\frac{1}{m}-2}+\frac{2 \alpha}{m} t^{\frac{1}{m}-1}\right) \\
\left(h^{\prime}\right)^{\prime} & \approx \frac{\alpha_{2}}{v} t^{\frac{1}{m}-2}+\beta_{2} t^{\frac{2}{m}-1} \\
\left(h^{\prime \prime}\right)^{\prime \prime} & \approx \alpha_{2} s^{\frac{1}{m}-2}+\beta_{2} s^{\frac{2}{m}-1} \\
h_{t t s s} & \sim\left(1+\frac{1}{m}\right) \alpha_{1} t^{\frac{1}{m}-2} \\
\left(h^{\prime}\right)^{\prime \prime} & \sim \frac{\alpha}{m} t^{\frac{1}{m}-1}
\end{aligned}
$$

also

$$
\begin{aligned}
\frac{1}{\lambda^{2}} & \approx 1-s=v^{m} \\
\operatorname{tr}(h) & \approx v^{m} h^{\prime}+v^{2 m} h^{\prime \prime} \approx \alpha v^{m-1} t^{\frac{1}{m}-1}+\beta t^{\frac{2}{m}-1}+\alpha s^{\frac{1}{m}-1} \\
\operatorname{det}(h) & \approx v^{3 m} h^{\prime} h^{\prime \prime} \approx \alpha^{2} t^{\frac{1}{m}-1} s^{\frac{1}{m}-1}+\alpha \beta t^{\frac{1}{m}-1} s^{\frac{2}{m}-1}
\end{aligned}
$$

As in the first case, we have

$$
M^{(1)} \approx v^{m}\left[\begin{array}{ccc}
t\left(h_{t}^{\prime}\right)^{2} & \bar{x}^{2} y^{2} h_{t}^{\prime} h_{t s s} & \bar{x} y h_{t}^{\prime} h_{s}^{\prime} \\
* & s\left(h_{s}^{\prime \prime}\right)^{2}+t s^{2}\left(h_{t s s}\right)^{2} & x \bar{y}\left(h_{t}^{\prime \prime} h_{s}^{\prime \prime}+s h_{s}^{\prime} h_{t s s}\right) \\
* & * & s\left(h_{s}^{\prime}\right)^{2}
\end{array}\right]
$$




$$
\begin{aligned}
& M^{(2)} \approx {\left[\begin{array}{ccc}
\frac{t}{|a|^{2}} h^{\prime} h_{t}^{\prime} & \frac{\bar{x}^{2} y^{2}}{|a|^{2}}\left(h^{\prime} h_{t s s}+\left(v^{m} h_{t s}-h^{\prime}\right) h_{s}^{\prime \prime}\right) \\
\frac{x^{2} \bar{y}^{2}}{|b|^{2}} h_{t s} h_{t}^{\prime} & \frac{s}{|b|^{2}}\left(v^{m} h^{\prime \prime} h_{s}^{\prime \prime}+t s h_{t s} h_{t s s}\right) \\
0 & 0 & \frac{\bar{x} y}{|a|^{2}} h^{\prime} h_{s}^{\prime} \\
\frac{x \bar{y}}{|b|^{2}}\left(v^{m} h^{\prime \prime} h_{t}^{\prime \prime}+s h_{t s} h_{s}^{\prime}\right) & 0
\end{array}\right] } \\
& M^{(3)} \approx v^{-m}\left[\begin{array}{ccc}
\frac{t}{|a|^{4}}\left(h^{\prime}\right)^{2} & \frac{\bar{x}^{2} y^{2}}{|a|^{2}|b|^{2}} h^{\prime} h_{t s} & 0 \\
* & \frac{s}{|b|^{4}}\left(v^{m}\left(h^{\prime \prime}\right)^{2}+t s h_{t s}^{2}\right) & 0 \\
0 & 0 & 0
\end{array}\right]
\end{aligned}
$$

Hence

$$
\begin{gathered}
U M^{(2)} \approx-\left[\begin{array}{ccc}
2 t h^{\prime} h_{t}^{\prime} & 2 \bar{x}^{2} y^{2}\left(h^{\prime} h_{t s s}+\left(v^{m} h_{t s}-h^{\prime}\right) h_{s}^{\prime \prime}\right) \\
2 x^{2} \bar{y}^{2} h_{t s} h_{t}^{\prime} & 2 s\left(v^{m} h^{\prime \prime} h_{s}^{\prime \prime}+t s h_{t s} h_{t s s}\right) \\
x \bar{y} h^{\prime} h_{t}^{\prime} & \bar{x} y\left(s h^{\prime} h_{t s s}+v^{m} h^{\prime \prime} h_{s}^{\prime \prime}\right) & \\
& 2 x \bar{y}\left(v^{m} h^{\prime \prime} h_{t}^{\prime \prime}+s h_{t s} h_{s}^{\prime}\right) \\
s h^{\prime} h_{s}^{\prime}
\end{array}\right] \\
U M^{(3)} U^{*} \approx v^{-m}\left[\begin{array}{ccc}
4 t\left(h^{\prime}\right)^{2} & 4 \bar{x}^{2} y^{2} h^{\prime} h_{t s} & 2 \bar{x} y\left(h^{\prime}\right)^{2} \\
* & 4 s\left(v^{m}\left(h^{\prime \prime}\right)^{2}+t s h_{t s}^{2}\right) & 2 x \bar{y}\left(v^{m}\left(h^{\prime \prime}\right)^{2}+s h^{\prime} h_{t s}\right) \\
* & * & s\left(h^{\prime}\right)^{2}
\end{array}\right]
\end{gathered}
$$

So $\hat{M}$ becomes

$$
\hat{M} \approx\left[\begin{array}{ccc}
v^{m} t\left(h_{t}^{\prime}\right)^{2} & \bar{x}^{2} y^{2} h_{t}^{\prime}\left(h_{t s s}-2 h_{t s}\right) & \\
* & s\left(h_{s}^{\prime \prime}\right)^{2}+t s^{2}\left(h_{t s s}-2 h_{t s}\right)^{2} & \\
* & * & \\
& & x y h_{t}^{\prime}\left(v^{m} h_{s}^{\prime}-h^{\prime}\right) \\
& & x \bar{y}\left[h_{t}^{\prime \prime} h_{s}^{\prime \prime}+s\left(h_{s}^{\prime}-h^{\prime}\right)\left(h_{t s s}-2 h_{t s}\right)\right] \\
& s\left(h_{s}^{\prime}-h^{\prime}\right)^{2}
\end{array}\right]
$$

(Here we only need the second leading terms for the $\hat{c}_{1}$ and $\hat{d}_{2}$ positions, as there will be cancellation later on.) 
Similarly,

$$
\begin{gathered}
N^{(1)} \approx v^{3 m}\left[\begin{array}{ccc}
t h^{\prime \prime}\left(h_{t}^{\prime}\right)^{2} & \bar{x}^{2} y^{2}\left[h_{t}^{\prime} h_{s}^{\prime \prime}\left(h^{\prime}-h_{t s}\right)+h^{\prime \prime} h_{t}^{\prime} h_{t s s}\right] & \bar{x} y h^{\prime \prime} h_{t}^{\prime} h_{s}^{\prime} \\
* & s h^{\prime}\left(h^{\prime \prime}\right)^{2}+t s^{2} h^{\prime \prime}\left(h_{t s s}\right)^{2} & x \bar{y} h^{\prime} h_{t}^{\prime \prime} h_{s}^{\prime \prime} \\
* & * & s h^{\prime \prime}\left(h_{s}^{\prime}\right)^{2}
\end{array}\right] \\
N^{(2)} \approx v^{2 m}\left[\begin{array}{ccc}
\frac{t}{|a|^{2}} h^{\prime} h^{\prime \prime} h_{t}^{\prime} & \frac{\bar{x}^{2} y^{2}}{|a|^{2}} h^{\prime} h^{\prime \prime} h_{t s s} & \frac{\bar{x} y}{|a|^{2}} h^{\prime} h^{\prime \prime} h_{s}^{\prime} \\
\frac{x^{2} \bar{y}^{2}}{|b|^{2}} h^{\prime} h^{\prime \prime} h_{t t s} & \frac{s}{|b|^{2}} h^{\prime} h^{\prime \prime} h_{s}^{\prime \prime} & \frac{x \bar{y}}{|b|^{2}} h^{\prime} h^{\prime \prime} h_{t}^{\prime \prime} \\
0 & 0 & 0
\end{array}\right] \\
N^{(3)} \approx v^{m}\left[\begin{array}{ccc}
\frac{t}{|a|^{4}}\left(h^{\prime}\right)^{2} h^{\prime \prime} & \frac{\bar{x}^{2} y^{2}}{|a|^{2}|b|^{2}} h^{\prime} h^{\prime \prime} h_{t s} & 0 \\
* & \frac{s}{|b|^{4}} h^{\prime}\left(h^{\prime \prime}\right)^{2} & 0 \\
0 & 0 & 0
\end{array}\right]
\end{gathered}
$$

Hence

$$
\begin{gathered}
U N^{(2)} \approx-v^{2 m}\left[\begin{array}{ccc}
2 t h^{\prime} h^{\prime \prime} h_{t}^{\prime} & 2 \bar{x}^{2} y^{2} h^{\prime} h^{\prime \prime} h_{t s s} & 2 \bar{x} y h^{\prime} h^{\prime \prime} h_{s}^{\prime} \\
2 x^{2} \bar{y}^{2} h^{\prime} h^{\prime \prime} h_{t t s} & 2 s h^{\prime} h^{\prime \prime} h_{s}^{\prime \prime} & 2 x \bar{y} h^{\prime} h^{\prime \prime} h_{t}^{\prime \prime} \\
x \bar{y} h^{\prime} h^{\prime \prime} h_{t}^{\prime} & \bar{x} y h^{\prime} h^{\prime \prime}\left(h_{s}^{\prime \prime}+s h_{t s s}\right) & s h^{\prime} h^{\prime \prime} h_{s}^{\prime}
\end{array}\right] \\
U N^{(3)} U^{*} \approx v^{m}\left[\begin{array}{ccc}
4 t\left(h^{\prime}\right)^{2} h^{\prime \prime} & 4 \bar{x}^{2} y^{2} h^{\prime} h^{\prime \prime} h_{t s} & 2 \bar{x} y\left(h^{\prime}\right)^{2} h^{\prime \prime} \\
* & 4 s h^{\prime}\left(h^{\prime \prime}\right)^{2} & 2 x \bar{y} h^{\prime} h^{\prime \prime}\left(h^{\prime \prime}+s h_{t s}\right) \\
* & * & s\left(h^{\prime}\right)^{2} h^{\prime \prime}
\end{array}\right]
\end{gathered}
$$

So the entries of $\hat{N}$ are

$$
\begin{aligned}
\hat{c}_{1}^{\prime} & \approx v^{3 m} t h^{\prime \prime}\left(h_{t}^{\prime}\right)^{2} \\
\hat{c}_{2}^{\prime} & \approx v^{3 m} s h^{\prime}\left(h_{s}^{\prime \prime}\right)^{2} \\
\hat{c}_{3}^{\prime} & \sim s^{\prime \prime}\left(h_{s}^{\prime}-h^{\prime}\right)^{2} \sim \alpha \alpha_{1}^{2} s^{\frac{1}{m}} t^{\frac{2}{m}-2} \\
\hat{d}_{1}^{\prime} & \sim \bar{x}^{2} y^{2}\left[h^{\prime \prime} h_{t}^{\prime} h_{t s s}+\left(h^{\prime} h_{t t s}-h_{t s} h_{t}^{\prime}\right) h_{s}^{\prime \prime}-2 h^{\prime} h^{\prime \prime} h_{t t s}\right] \\
& \sim \bar{x}^{2} y^{2}\left[\alpha \alpha_{1}^{2} t^{\frac{2}{m}-3} s^{\frac{1}{m}-1}+o\left(t^{\frac{1}{m}-2} s^{\frac{2}{m}-3}\right)\right] \\
\hat{d}_{2}^{\prime} & \approx \bar{x} y\left(v^{m} h_{s}^{\prime}-h^{\prime}\right) v^{2 m} h^{\prime \prime} h_{t}^{\prime} \sim \bar{x} y \alpha \alpha_{1}^{2} t^{\frac{2}{m}-3} s^{\frac{1}{m}-1} \\
\hat{d}_{3}^{\prime} & \sim x \bar{y} h^{\prime} h_{t}^{\prime \prime} h_{s}^{\prime \prime} \sim x \bar{y} \alpha^{2} \alpha_{1} t^{\frac{2}{m}-2} s^{\frac{1}{m}-2}
\end{aligned}
$$


Finally, $H^{(1)}, H^{(2)}$ and $H^{(3)}$ are given in the first case, and we have

$$
\begin{gathered}
U H^{(2)} \approx-v^{-m}\left[\begin{array}{ccc}
2 t h_{t}^{\prime} & 2 \bar{x}^{2} y^{2} h_{t s s} & 2 \bar{x} y h_{s}^{\prime} \\
2 x^{2} \bar{y}^{2} h_{t t s} & 2 s h_{s}^{\prime \prime} & 2 x \bar{y} h_{t}^{\prime \prime} \\
x \bar{y} h_{t}^{\prime} & \bar{x} y\left(h_{s}^{\prime \prime}+s h_{t s s}\right) & s h_{s}^{\prime}
\end{array}\right] \\
U H^{(3)} U^{*} \approx v^{-2 m}\left[\begin{array}{ccc}
4 t h^{\prime} & 4 \bar{x}^{2} y^{2} h_{t s} & 2 \bar{x} y h^{\prime} \\
* & 4 s h^{\prime \prime} & 2 x \bar{y}\left(h^{\prime \prime}+s h_{t s}\right) \\
* & * & s h^{\prime}
\end{array}\right]
\end{gathered}
$$

Therefore the entries of $\hat{H}$ are

$$
\begin{aligned}
& \hat{e}_{1} \approx e_{1}^{(1)}=\left(h^{\prime}\right)^{\prime} \approx \frac{\alpha_{2}}{v} t^{\frac{1}{m}-2}+\beta_{2} t^{\frac{2}{m}-2} \\
& \hat{e}_{2} \approx e_{2}^{(1)}=\left(h^{\prime \prime}\right)^{\prime \prime} \approx \alpha_{2} s^{\frac{1}{m}-2}+\beta_{2} s^{\frac{2}{m}-2} \\
& \hat{e}_{3} \sim e_{3}^{(1)}=\left(h^{\prime}\right)^{\prime \prime} \sim \frac{\alpha}{m} t^{\frac{1}{m}-1} \\
& \hat{f}_{1} \sim \bar{x}^{2} y^{2}\left(h_{t t s s}-2 h_{t t s}\right) \sim \bar{x}^{2} y^{2} \alpha_{2} t^{\frac{1}{m}-2} \\
& \hat{f}_{2} \approx \bar{x} y\left(h_{t s}^{\prime}-v^{-m} h_{t}^{\prime}\right) \sim \bar{x} y \alpha_{2} t^{\frac{1}{m}-2} \\
& \hat{f}_{3} \sim x \bar{y}\left(h_{t s}^{\prime \prime}-2 h_{t}^{\prime \prime}-h_{s}^{\prime \prime}\right) \sim x \bar{y}\left(\alpha_{2} s^{\frac{1}{m}-2}+2 \alpha_{1} t^{\frac{1}{m}-1}\right)
\end{aligned}
$$

By comparing the leading orders, we know that $\hat{e}_{i}>0, \hat{e}_{1} \hat{e}_{2} \gg \hat{e}_{1} \gg \hat{e}_{3}^{2}$, $\hat{e}_{3} \gg\left|\hat{f}_{1}\right|$, and $\hat{e}_{1} \hat{e}_{3} \gg\left|\hat{f}_{2}\right|^{2}, \hat{e}_{2} \hat{e}_{3} \gg\left|\hat{f}_{3}\right|^{3}$. Hence $Q_{\hat{H}}>0$, and $Q_{1}=\operatorname{tr}(h) Q_{G}+Q_{\hat{H}}>0$.

Next, consider $\operatorname{tr}(h) \hat{H}-\hat{M}$, since

$$
\begin{aligned}
\operatorname{tr}(h) \hat{e}_{1}-\hat{c}_{1} & \approx \operatorname{tr}(h)\left(h^{\prime}\right)^{\prime}-v^{m} t\left(h_{t}^{\prime}\right)^{2} \\
& \sim \frac{1}{m^{2}} \alpha \beta t^{\frac{3}{m}-3}+\alpha \alpha_{2} t^{\frac{1}{m}-2} s^{\frac{1}{m}-1}>0 \\
\operatorname{tr}(h) \hat{e}_{2}-\hat{c}_{2} & \sim \alpha \alpha_{2} t^{\frac{1}{m}-1} s^{\frac{1}{m}-2}>0 \\
\operatorname{tr}(h) \hat{e}_{3}-\hat{c}_{3} & \sim \frac{1}{m} \alpha^{2} t^{\frac{2}{m}-2}>0 \\
\operatorname{tr}(h) \hat{f}_{1}-\hat{d}_{1} & \sim-\bar{x}^{2} y^{2}\left[\alpha_{1}^{2} t^{\frac{1}{m}-2} s^{\frac{1}{m}-2}+o\left(t^{\frac{2}{m}-3}\right)\right] \\
\operatorname{tr}(h) \hat{f}_{2}-\hat{d}_{2} & \approx \bar{x} y\left[\operatorname{tr}(h)\left(h_{t s}^{\prime}-v^{-m} h_{t}^{\prime}\right)-h_{t}^{\prime}\left(v^{m} h_{s}^{\prime}-h^{\prime}\right)\right]
\end{aligned}
$$




$$
\begin{aligned}
& \sim \bar{x} y\left(\frac{\alpha \beta}{m^{2}} t^{\frac{3}{m}-3}+\alpha \alpha_{1} t^{\frac{1}{m}-2} s^{\frac{1}{m}-1}\right) \\
\operatorname{tr}(h) \hat{f}_{3}-\hat{d}_{3} & \sim x \bar{y}\left(2 \alpha \alpha_{1} t^{\frac{2}{m}-2}+\left(\frac{1}{m}-2\right) \alpha \alpha_{1} t^{\frac{1}{m}-1} s^{\frac{1}{m}-2}\right)
\end{aligned}
$$

So the entries of the matrix $\operatorname{tr}(h) \hat{H}-\hat{M}$ satisfy

$$
\begin{aligned}
A & \sim t^{\frac{3}{m}-3}+t^{\frac{1}{m}-2} s^{\frac{1}{m}-1} \\
B & \sim t^{\frac{2}{m}-2} \\
C & \sim t^{\frac{1}{m}-1} s^{\frac{1}{m}-2} \\
|D| & \leq t^{\frac{1}{m}-1} s^{\frac{1}{m}-1}+o\left(s t^{\frac{2}{m}-2}\right) \\
|E| & \leq \sqrt{t s}\left(t^{\frac{3}{m}-3}+t^{\frac{1}{m}-2} s^{\frac{1}{m}-1}\right) \\
|F| & \leq \sqrt{t s}\left(t^{\frac{1}{m}-1} s^{\frac{1}{m}-2}+t^{\frac{2}{m}-2}\right)
\end{aligned}
$$

Here again we ignored the positive constant coefficients. Therefore, it is easy to check that

$$
A>0, B>0, C>0, A C \gg B^{2} \gg|D|^{2}, A B \gg|E|^{2}, B C \gg|F|^{2}
$$

Hence $Q_{2}>0$.

Similarly, for the matrix $\operatorname{det}(h) \hat{H}-\hat{N}$, we compute

$$
\begin{aligned}
\operatorname{det}(h) \hat{e}_{1}-\hat{c}_{1}^{\prime} & \approx v^{3 m} h^{\prime \prime}\left[h^{\prime}\left(h^{\prime}\right)^{\prime}-t\left(h_{t}^{\prime}\right)^{2}\right] \sim \frac{1}{m^{2}} \alpha \beta t^{\frac{3}{m}-3} s^{\frac{1}{m}-1}>0 \\
\operatorname{det}(h) \hat{e}_{2}-\hat{c}_{2}^{\prime} & \approx v^{3 m} h^{\prime}\left[h^{\prime \prime}\left(h^{\prime \prime}\right)^{\prime \prime}-s\left(h_{s}^{\prime \prime}\right)^{2}\right] \sim \frac{1}{m^{2}} \alpha^{2} \beta t^{\frac{1}{m}-1} s^{\frac{3}{m}-3}>0 \\
\operatorname{det}(h) \hat{e}_{3}-\hat{c}_{3}^{\prime} & \sim \frac{1}{m} \alpha^{3} t^{\frac{2}{m}-2} s^{\frac{1}{m}-1}>0 \\
\operatorname{det}(h) \hat{f}_{1}-\hat{d}_{1}^{\prime} & \sim-\bar{x}^{2} y^{2}\left[o\left(t^{\frac{2}{m}-3} s^{\frac{1}{m}-1}\right)+o\left(t^{\frac{1}{m}-2} s^{\frac{2}{m}-3}\right)\right] \\
\operatorname{det}(h) \hat{f}_{2}-\hat{d}_{2}^{\prime} & \approx v^{3 m} h^{\prime} h^{\prime \prime} \cdot \bar{x} y\left(h_{t s}^{\prime}-v^{-m} h_{t}^{\prime}\right)-\bar{x} y\left(v^{m} h_{s}^{\prime}-h^{\prime}\right) v^{2 m} h^{\prime \prime} h_{t}^{\prime} \\
& \sim \bar{x} y h^{\prime \prime}\left(\frac{1}{m^{2}} \alpha \beta t^{\frac{3}{m}-3}+\alpha \alpha_{1} t^{\frac{1}{m}-2} s^{\frac{1}{m}-1}\right) \\
\operatorname{det}(h) \hat{f}_{3}-\hat{d}_{3}^{\prime} & \sim x \bar{y}\left(\alpha^{2} \alpha_{2} t^{\frac{1}{m}-1} s^{\frac{2}{m}-3}-\alpha^{2} \alpha_{1} t^{\frac{2}{m}-2} s^{\frac{1}{m}-1}\right)
\end{aligned}
$$


Hence the entries of the matrix $\operatorname{det}(h) \hat{H}-\hat{N}$ satisfy

$$
\begin{aligned}
A & \sim t^{\frac{3}{m}-3} s^{\frac{1}{m}-1} \\
C & \sim t^{\frac{1}{m}-1} s^{\frac{3}{m}-3} \\
B & \sim t^{\frac{2}{m}-2} s^{\frac{1}{m}-1} \\
|D| & \ll t^{\frac{2}{m}-2} s^{\frac{1}{m}}+t^{\frac{1}{m}-1} s^{\frac{2}{m}-2} \\
|E| & \leq \sqrt{t s}\left(t^{\frac{3}{m}-3} s^{\frac{1}{m}-1}+t^{\frac{1}{m}-2} s^{\frac{2}{m}-2}\right) \\
|F| & \leq \sqrt{t s}\left(t^{\frac{1}{m}-1} s^{\frac{2}{m}-3}+t^{\frac{2}{m}-2} s^{\frac{1}{m}-2}\right)
\end{aligned}
$$

Therefore, these entries satisfy

$$
A>0, B>0, C>0, A C \gg B^{2} \gg|D|^{2}, A B \gg|E|^{2}, B C \gg|F|^{2}
$$

Hence $Q_{3}>0$. Thus we have completed the proof of Theorem 2 .

Remark. In the general case when the two multiplicities $m_{1}$ and $m_{2}$ are not equal or the two branches $D_{1}, D_{2}$ are not perpendicular to each other at the node $q_{0}$, the proof is similar but more tedious. We omit the details here. The most complicated part in the explicit computation of the asymptotic expansions is of course when the leading terms cancelling each others and one has to go to the next leading term. Especially in $A, B, C$, one needs their leading coefficients to be positive. However, since locally, away from $R$, the metric $\omega_{\epsilon}$ is the sum of two metrics, each of them is negatively curved by Theorem 1 , so we know that the sum metric $\omega_{\epsilon}$ at least has negative bisectional curvature. Hence all the $A, B, C$ terms should have the right sign.

When the dimension is higher than two, we have checked that the same metric is still quasi- negatively curved (in the strongest sense). However, at this moment we don't know of any examples in dimensions $n>2$ of good coverings over a compact ball quotient with totally geodesic branching locus. When $n=2$, there are three sequences of such surfaces constructed by Hirzebruch $([\mathrm{H}])$. We shall discuss them in the next section. 


\section{Examples.}

Let us recall Hirzebruch's surfaces constructed from arrangements of lines. Let $\varphi: \mathbf{P}^{2} \rightarrow \mathbf{P}^{k}$ be a linear embedding defined by $k+1$ linear forms $l_{0}, \ldots, l_{k}$. Let $f_{n}: \mathbf{P}^{k} \rightarrow \mathbf{P}^{k}$ be the map defined by $f\left(\left[x_{0}: \ldots: x_{k}\right]\right)=$ $\left[x_{0}^{n}: \ldots: x_{k}^{n}\right]$. Then the inverse image $X_{n}=f_{n}^{-1}\left(\varphi\left(\mathbf{P}^{2}\right)\right)$ is an irreducible normal surface, with singular point set $S$ being the inverse image of $S_{0}$, the set of points in $\mathbf{P}^{2}$ where there are more than two $l_{i}$ 's pass through it. Blowing up points of $S$ in $\mathbf{P}^{k}$ will give a smooth resolution $Y_{n}$ of $X_{n}$. Let $D=D^{(n)}$ be the union of the exceptional curves and those curves above the lines $l_{i}$ 's.

When $m \mid n$, one has good coverings $Y_{n} \rightarrow Y_{m}$, with branching locus $D^{(m)}$ and ramification locus $D^{(n)}$ (each curve has multiplicity $\frac{n}{m}$ ).

Hirzebruch computed the Chern numbers of $Y_{n}$ in terms of the combinatorial data of the line arrangement $\Lambda=\left\{l_{0}, \ldots, l_{k}\right\}$. He found that there are three cases where $c_{1}^{2}=3 c_{2}$, (and $c_{1}<0$ ), hence by Yau's Theorem $([\mathrm{Y}])$, these three surfaces are complex ball quotients. The corresponding divisor $D$ is totally geodesic, since its components are pointwisely fixed by some deck transformations of the covering $h_{n}: Y_{n} \rightarrow \hat{\mathbf{P}}^{2}$, where $\hat{\mathbf{P}}^{2}$ is the blowing up of $\mathbf{P}^{2}$ at the points in $S_{0}$; while the canonical metric for a ball quotient is invariant under any biholomorphism, so the curve $D$ must be totally geodesic with respect to the canonical metric.

The three surfaces are:

1). $Y_{5}(A)$, where $A$ is the arrangement of 6 lines in $\mathbf{P}^{2}$ defined by $\left\{x_{i}, 0 \leq\right.$ $\left.i \leq 2 ; x_{i}-x_{j}, 0 \leq i<j \leq 2\right\}$ in the homogeneous coordinate $\left[x_{0}: x_{1}: x_{2}\right]$ of $\mathbf{P}^{2}$. It is a real simplicial arrangement.

2). $Y_{3}(B)$, where $B$ is the 12 lines in a Hesse pencil (fix a smooth cubic curve $\Sigma$ in $\mathbf{P}^{2}$, let $S$ be the nine reflection points of $\Sigma$, then all the cubics containing $S$ forms a pencil. There are exactly 4 degenerating curves in that pencil, each is the union of three lines. $B$ is just those 12 lines).

3). $Y_{5}(C)$, where $C$ is the arrangement of 9 lines coming from the nine reflection points of a smooth plane cubic by dualizing.

Lemma. For each of the three surfaces above, the divisor $D$ is not only totally geodesic and normal crossing, but also intersect perpendicularly at all the nodes.

Proof. For the perpendicularity, let $p$ be a node of $D$, and $D_{1}, D_{2}$ be the two branches of $D$ passing through $p$. In each case, there always exists a 
biholomorphism $f$ of the surface such that $f$ fixs both $D_{i}$, and $d f_{p}$ is not a scalar multiple of the identity. (We leave this verification to the reader). Now the two tangent directions of $D_{i}$ at $p$ are eigenvectors of $d f_{p} \in U(2)$, which is not a scalar multiple of the identity, so they must be perpendicular to each other.

In $[\mathrm{H}]$, Hirzebruch also computed the ratio of the two Chern numbers: For $Y=Y_{5 n}(A)$, its

$$
\frac{c_{1}^{2}}{c_{2}}=\frac{5}{2}+\frac{10 n-7}{2\left(10 n^{2}-10 n+3\right)}
$$

For $Y=Y_{3 n}(B)$,

$$
\frac{c_{1}^{2}}{c_{2}}=\frac{5}{2}+\frac{27 n-19}{8\left(7 n^{2}-7 n+2\right)}
$$

For $Y=Y_{5 n}(C)$,

$$
\frac{c_{1}^{2}}{c_{2}}=\frac{8}{3}+\frac{100 n-63}{3\left(75 n^{2}-50 n+12\right)}
$$

All three sequences are monotonicly decreasing in $n$.

By Theorem 2, we know that these three sequences of surfaces all admit Kähler metrics with quasi-negative complex curvature operator. In particular, they are all strongly rigid.

Let us conclude this section by giving a couple of remarks on an observation of Enoki, which gives an alternative way to detect the totally geodesy of a smooth curve $C$ in a ball quotient $X^{2}$. His result is the following (cf. [H1], p.142):

Any smooth curve $C$ in a compact ball quotient $X^{2}$ satisfies the inequality $K_{X} C \geq-3 C^{2}$, and the equality holds when and only when $C$ is totally geodesic (with respect to the canonical metric of $X$ ).

We remark here that the same is true when $X^{2}$ is non-compact but of finite volume and $C$ touchs the infinity. To be more precise, one has:

Lemma. Let $X^{2}$ be a smooth finite volume ball quotient. Let $\bar{X}$ be any smooth compactification of $X$ such that $E=\bar{X} \backslash X$ is a normal crossing divisor. Then for any smooth curve $C$ in $\bar{X}$ which is not contained in $E, C$ satisfies

$$
\left(K_{\bar{X}}+E\right) C \geq-3 C^{2}
$$

with equality holds when and only when $C+E$ is normal crossing and $C \cap X$ is totally geodesic in $X$. 
Proof. Let $\left\{e_{1}, e_{2}\right\}$ be an unitary frame along $C_{0}=C \cap X$ such that $e_{1}$ is tangential to $C$. Then by the Cohn-Vossen equality ([Hu], p.58, Theorem 11, the conditions are satisfied because for the punctured disc with Poincare metric $h$, the circles centered at the origin with (euclidean) radius $r$ will have $h$-length goes to zero when $r \rightarrow 0$, then one can use the Schwartz lemma), one has

$$
e\left(C_{0}\right)=\frac{1}{2 \pi} \int_{C_{0}} R_{1 \overline{1} 1 \overline{1}}\left(\left.g\right|_{C_{0}}\right)
$$

Here $e$ denote the Euler number and $g$ is the canonical metric of $X$. Since $R_{1 \overline{1} 1 \overline{1}}\left(\left.g\right|_{C_{0}}\right) \leq R_{1 \overline{1} 1 \overline{1}}(g)$, where the difference is the square of the norm of the second fundamental form (so equality holds identically when and only when $C_{0}$ is totally geodesic), and $R_{1 \overline{1} 1 \overline{1}}(g)=2 R_{1 \overline{1} 2 \overline{2}}(g)=\frac{3}{2} R i c c i_{1 \overline{1}}(g)$, we get

$$
-\left(K_{\bar{X}}+E\right) C=\frac{1}{2 \pi} \int_{C_{0}} \operatorname{Ricci}_{1 \overline{1}}(g) \geq \frac{3}{2} e\left(C_{0}\right) \geq \frac{3}{2}(e(C)-C E)
$$

where the last inequality becomes equality only when $C+E$ is normal crossing. From this one gets the desired inequality, with equality holds when $C+E$ is normal crossing and $C_{0}$ is totally geodesic.

An example of such kind is again given by Hirzebruch in [H2], where $E$ is the disjoint union of a bunch of elliptic curves, and $C$ is one of the $L_{j}$ in his paper. The intersection numbers are:

$$
C^{2}=-n^{3}, C E=4 n^{2}, e(C)=-2 n^{3}+4 n^{2}
$$

so $(K+E) C=-e(C)-C^{2}+C E=3 n^{3}$. Therefore, from the above lemma, we know that $C_{0}$ is totally geodesic in the non-compact ball quotient $X$.

Of course one can also use the deck transformation and the invariance of the canonical metric to show this.

By using the fact that the bisectional curvature of a complex submanifold is always smaller than or equal (when totally geodesic) to the curvature of the ambient manifold, together with the nice curvature behavior of the complex ball, we know that the high dimensional case of Enoki's result is also true:

Lemma. If $X^{n}$ is a smooth compact ball quotient of finite volume. Let $\bar{X}$ be a smooth compactification of $X$ with $D=\bar{X} \backslash X$ a normal crossing divisor. Assume $C$ is a smooth divisor in $\bar{X}$ such that $C$ is not contained in $D$ and the second fundamental form of $C \cap X$ in $X$ has bounded norm. Then for any integer $1 \leq r<n$, it holds:

$$
(K+C+D)^{r} \cdot(K+D)^{n-1-r} \cdot C \geq\left(\frac{n}{n+1}\right)^{r}(K+D)^{n-1} \cdot C
$$


where $K=K_{\bar{X}}$ is the canonical divisor. If any one of the above is an equality, then $C \cap X$ is totally geodesic in $X$. In this case all the above become equalities.

\section{Non-compact case.}

We would also like to construct non-compact, finite volume complete Kähler manifolds with negative complex curvature operator. The previously known examples are those (non-compact) ball quotients. We claim the following:

Theorem 3. If $X^{n}$ is a complex ball quotient with finite volume and $D$ is a smooth compact divisor in $X$ which is totally geodesic with respect to the canonical metric $g$ on $X$. Then the complement $Y=X \backslash D$ admits a complete Kähler metric with finite volume such that its complex curvature operator is everywhere negative definite and bounded from below.

Such $Y$ can not be biholomorphic to a ball quotient by Mumford's proportionality principle on the Chern numbers ([M]).

Proof. Let $g$ be the canonical metric of $X^{n}$, and $d$ be the Poincare distance function from $D$. Also let $\rho$ be a smooth non-decreasing function on $[0, \infty]$ such that $\rho(x)=x$ for $0 \leq x \leq \delta_{1}$ and $\rho$ is constant for $x \geq \delta_{2}$. Here $0<\delta_{1}<\delta_{2}$ are two sufficiently small constants. Then $d_{1}=\rho \circ d$ is a smooth function on $X$, and is positive in $Y$.

Consider the Poincare metric

$$
\omega_{h}=\sqrt{-1} \partial \bar{\partial} h, \quad h=3 \log \Phi-\log \left[d_{1}\left(\log d_{1}\right)^{2}\right]
$$

where $\Phi$ is the volume form of $g . h$ is a metric in a deleted neighborhood $U^{0}=U \backslash D$, where $U$ is a small tubular neighborhood of $D$.

Define

$$
\omega_{\epsilon}=\omega_{g}+\epsilon \omega_{h}
$$

It is not hard to see that $\omega_{\epsilon}$ is a complete Kähler metric of finite volume on $Y=X \backslash D$ for all sufficiently small $\epsilon>0$. We want to show that there exists a small neighborhood $U_{1}$ of $D$ such that, for all $0<\epsilon<\epsilon_{0}$, the complex curvature operator of $\omega_{\epsilon}$ is negative and bounded from below in $U_{1}^{0}=U_{1} \backslash D$. For this purpose, let us fix a point $p \in Y$ near $D$. Let $q \in D$ be the closest point to $p$. Again let $\left\{z_{1}, \ldots, z_{n}\right\}$ be the canonical coordinate of the complex unit ball so that $q$ is (underneath) the origin and $D$ is (underneath) the 
hyperplane $\left\{z_{1}=0\right\}$. Then $p$ has coordinate $(a, 0, \ldots, 0)$. Let $\varphi$ be the local isometry of $g$ defined by $\varphi\left(z_{1}, \ldots, z_{n}\right)=\left(w, z_{2}, \ldots, z_{n}\right)$ where

$$
w=\frac{z_{1}+a}{1+\bar{a} z_{1}}
$$

The curvature of the metric $\omega_{\epsilon}$ at $p$ is the same as the curvature of $\varphi^{*}\left(\omega_{\epsilon}\right)$ at $q$. Let us still denote this metric by $\omega_{g}+\epsilon \omega_{h}$, only now $h$ becomes

$h=-\log \left[(1-t-s)^{2} d(\log d)^{2}\right], \quad d=\frac{t}{1-s}, \quad t=|w|^{2}, s=\left|z_{2}\right|^{2}+\cdots\left|z_{n}\right|^{2}$

Here again we throw away the cut-off function $\rho$, as we are examining the situation very close to $D$.

We want to show that there exists $r>0$, independent of $\epsilon$, such that the above metric has the desired curvature property at the origin $q=0$ whenever $0<a \leq r$.

As in the proof of Theorem 1, since $h=h(t, s)$ and $s=0$ at $q=0$, so at the origin all the components of the curvature tensor vanish except those bisectional curvature terms $R_{i \bar{i} j \bar{j}}$. Denote it by $-a_{i j}$. Then the complex curvature operator is negative definite at $q$ if and only if the matrix $A=\left(a_{i j}\right)$ is positive definite. Again by the symmetry among $\left\{z_{2}, \ldots z_{n}\right\}, A$ takes the special form

$$
A=\left(a_{i j}\right)=\left[\begin{array}{cc}
b & c x^{*} \\
c x & e\left(I+x x^{*}\right)
\end{array}\right]
$$

where $x=(1, \ldots, 1)^{*}$ and

$$
\begin{aligned}
& b=2+\epsilon h_{1 \overline{1} 1 \overline{1}}-\epsilon^{2}\left|h_{1 \overline{1} 1}\right|^{2}\left(1+\epsilon h_{1 \overline{1}}\right)^{-1} \\
& c=1+\epsilon h_{1 \overline{1} 2 \overline{2}}-\epsilon^{2}\left|h_{2 \overline{2}}\right|^{2}\left(1+\epsilon h_{2 \overline{2}}\right)^{-1} \\
& e=1+\epsilon h_{2 \overline{2} 3 \overline{3}}
\end{aligned}
$$

So $A>0$ is equivalent to

$$
b>0, \quad e>0, \quad \text { and } n b e-(n-1) c^{2}>0
$$

Since $h=-\log \left[(1-t-s)^{3} d(\log d)^{2}\right]$, we compute the expansion when $t=|a|^{2} \rightarrow 0$ :

$$
\begin{aligned}
h_{2 \overline{2}} & \sim 2 \\
h_{2 \overline{2} 3 \overline{3}} & \sim 2 \\
h_{2 \overline{2} 1} & \sim \bar{a} \frac{2}{t(\log t)^{2}}
\end{aligned}
$$




$$
\begin{aligned}
h_{1 \overline{1} 2 \overline{2}} & \sim \frac{4}{t(-\log t)^{3}} \\
h_{1 \overline{1}} & \sim \frac{2}{t(\log t)^{2}} \\
h_{1 \overline{1} 1} & \sim-\frac{2 \bar{a}}{t^{2}(\log t)^{2}}\left(1+\frac{2}{\log t}\right) \\
h_{1 \overline{1} 1 \overline{1}} & \sim \frac{2}{t^{2}(\log t)^{2}}\left(1+\frac{4}{\log t}+\frac{6}{(\log t)^{2}}\right)
\end{aligned}
$$

Hence

$$
\begin{gathered}
R_{1} \equiv h_{1 \overline{1}} h_{1 \overline{1} 1 \overline{1}}-\left|h_{1 \overline{1} 1}\right|^{2} \sim \frac{8}{t^{3}(\log t)^{6}}>0 \\
R_{2} \equiv h_{2 \overline{2}} h_{1 \overline{1} 2 \overline{2}}-\left|h_{2 \overline{2} 1}\right|^{2} \sim \frac{4}{t(-\log t)^{3}}\left(2+\frac{1}{\log t}\right)>0
\end{gathered}
$$

Hence $c>0$. Since $0<c \leq c_{1} \equiv 1+\epsilon h_{1 \overline{1} 2 \overline{2}}$, so in order to show that $n b e>(n-1) c^{2}$, it suffices to that

$$
Q \equiv\left(1+\epsilon h_{1 \overline{1}}\right)\left[n b e-(n-1) c_{1}^{2}\right]>0
$$

But $Q=(n+1)+Q_{1} \epsilon+Q_{2} \epsilon^{2}+Q_{3} \epsilon^{3}$, with

$$
\begin{aligned}
& Q_{1}=n\left(h_{1 \overline{1} 1 \overline{1}}+2 h_{2 \overline{2} 3 \overline{3}}+2 h_{1 \overline{1}}\right)-(n-1)\left(h_{1 \overline{1}}+2 h_{1 \overline{1} 2 \overline{2}}\right) \sim n h_{1 \overline{1} 1 \overline{1}} \\
& Q_{2}=n\left[R_{1}+h_{2 \overline{2} 3 \overline{3}}\left(h_{1 \overline{1} 1 \overline{1}}+2 h_{1 \overline{1}}\right)\right]-(n-1) h_{1 \overline{1} 2 \overline{2}}\left(h_{1 \overline{1} 2 \overline{2}}+2 h_{1 \overline{1}}\right) \sim n R_{1} \\
& Q_{3}=n R_{1} h_{2 \overline{2} 3 \overline{3}}-(n-1) h_{1 \overline{1}}\left(h_{1 \overline{1} 2 \overline{2}}\right)^{2} \sim \frac{8 n}{t^{4}(\log t)^{6}}
\end{aligned}
$$

Hence all $Q_{i}>0$ and we have proved that $A>0$.

For the lower bound, denote by $\alpha=1+\epsilon h_{1 \overline{1}}, \quad \beta=1+\epsilon h_{2 \overline{2}}$, and let $\left\{e_{1}, \ldots, e_{n}\right\}$ be the natural basis at the origin. Write

$$
e_{1}^{\prime}=\frac{1}{\sqrt{\alpha}} e_{1}, \quad e_{i}^{\prime}=\frac{1}{\sqrt{\beta}} e_{i} \quad 2 \leq i \leq n
$$

Then $\left\{e_{i}^{\prime}\right\}$ becomes an unitary tangent frame for the metric $g+\epsilon h$. Let $-Q$ be the complex curvature operator of $g+\epsilon h$ at the origin. Under this unitary frame, $Q$ has the form

$$
Q=\left[\begin{array}{ccc}
A^{\prime} & & \\
& c^{\prime} I_{2 n-2} & \\
& & e^{\prime} I_{N}
\end{array}\right], \quad A^{\prime}=\left[\begin{array}{cc}
b^{\prime} & c^{\prime} x^{*} \\
c^{\prime} x & e^{\prime}\left(I_{n-1}+x x^{*}\right)
\end{array}\right]
$$


where $N=(n-1)(n-2), x=(1, \ldots 1)^{*}$, and

$$
b^{\prime}=\frac{b}{\alpha^{2}}, \quad c^{\prime}=\frac{c}{\alpha \beta}, \quad e^{\prime}=\frac{e}{\beta^{2}}
$$

The eigenvalues of $Q$ are therefore $c^{\prime}, e^{\prime}$, and $\lambda_{1,2}$ :

$$
\lambda_{1,2}=\frac{1}{2}\left(b^{\prime}+n e^{\prime}\right) \pm \frac{1}{2} \sqrt{\left(b^{\prime}-n e^{\prime}\right)^{2}+(n-1) c^{\prime 2}}
$$

So the curvature operator will be bounded from below if $b^{\prime}, c^{\prime}, e^{\prime}$ are all bounded from above. Since we have fixed $\epsilon$ now, it is straight forward to verify that they are indeed bounded when $t=|a|^{2} \rightarrow 0$.

It is also not hard to see that $c^{\prime} \rightarrow 0$ when $t \rightarrow 0$. This says that the curvature is not uniformly negative.

We have completed the proof of Theorem 3.

In [K-M], Kudla and Millson proved the existence in any dimension of the pair $\left(X^{n}, D\right)$ of smooth compact complex ball quotient with smooth totally geodesic divisor. So the above theorem implies:

Corollary. For any dimension n, there exists complete Kähler manifold $\left(Y^{n}, g\right)$ of finite volume whose complex curvature operator is negative and bounded from below, and $Y$ can not be biholomorphic (or even properly homotopic) to any locally hermitian symmetric manifolds.

Let us conclude this section by making a couple of remarks about the Theorem 3.

Remark. In Theorem 3, if $D$ has more than one components, then the same conclusion still holds. But the computation is more tedious. In another case, if $X$ is non-compact and $D$ touches the infinity, then the complement is still negatively curved. However, in this situation, one needs to add a contribution term from each infinity torus to the metric $\omega_{\epsilon}$, as the injectivity radius goes down to zero at infinity. The computation is possible by the nice explicit expression of the complex hyperbolic metric in the coordinate neighborhood at the infinity, given by Mok in [Mo], where he treated both the arithmetic case and the non-arithmetic case.

It is perhaps worth mentioning that in the example of [H2], the totally geodesic divisor $C$ and the infinity divisor $E$ satisfy $(E+C)^{2}=0$. So the curve $E+C$ can not be blown down to a singular point. So by the result of Siu-Yau [S-Y], the complement $Y=X \backslash C=\bar{X} \backslash(E \cup C)$ can not 
admit any complete Kähler metric of finite volume with sectional curvature bounded between two negative constants. So in order to improve the result of Theorem 3 to get uniformly negative curvature, one has to take into consideration the (global) information that the divisors to be deleted are resolutions to isolated singularities.

There are some related problems that can be studied. For instance, let $\left(X^{n}, D\right)$ be a Kudla-Millson pair of ball quotient with smooth totally geodesic divisor. Then what can we say about the universal covering space of $X \backslash D$ ? Is it sometimes a bounded domain?

\section{References.}

[C-W] C.-K. Cheung and H. Wu, Some new domains with complete Kähler metrics of negative curvature, J. of Geometric Analysis 2 (1992), 37-78.

[G-T] M. Gromov and W. Thurston, Pinching constants for hyperbolic manifolds, Invent. Math. 89 (1987), 1-12.

[H] F. Hirzebruch, Arrangements of lines and algebraic surfaces, Arithmetic and Geometry, vol II. Progress in Math, vol 36, pp. 113-140. Boston, Basel, Stuttgart: Birkhäuser 1983.

[H1] F. Hirzebruch, Algebraic surfaces with extreme Chern numbers (report on the thesis of Th. Höfer, Bonn 1984), Uspekhi Mat. Nauk 40:4 (1985), 121129.

[H2] F. Hirzebruch, Chern numbers of algebraic surfaces, Math. Ann. 266 (1984), $351-356$.

[Hu] A. Huber, On subharmonic functions and differential geometry in the large, Comm. Math. Helv. 32 (1957), 13-72.

[K-M] S. Kudla and J. Millson, Intersection numbers of cycles on locally symmetric spaces and Fourier coefficients of holomorphic modular forms in several complex variables, Publ. Math. I.H.E.S. 71 (1990), 121-172.

[M] D. Mumford, Hirzebruch's proportionality theorem in the non-compact case, Invent. Math. 42 (1977), 239-272.

[Mo] N-M Mok, Local biholomorphisms between hermitian locally symmetric spaces of non-compact type, preprint.

[M-S] G.D. Mostow and Y-T Siu, A compact Kähler surface of negative curvature not covered by the ball, Annals Math. 112 (1980), 321-360. 
[S-Y] Y-T Siu and S-T Yau, Compactification of negatively curved complete Kähler manifolds of finite volume, in Seminar on Differential Geometry, ed. by Yau, Annals of Math. Studies, vol.102, Princeton Univ. Press, Princeton, 1982, 363-380.

[Y] S-T Yau, Calabi's conjecture and some new results in algebraic geometry, Proc. Nat. Acad. Sci. USA 74 (1977), 1798-1799.

[Z] F. Zheng, Intersection domains and the sum of two complex hyperbolic metrics, to appear in J. of Geometric Analysis.

[Z1] F. Zheng, First Pontrjagin form, rigidity and strong rigidity of nonpositively curved Kähler surface of general type, to appear in Math. Zeit.

ReCeived JANUARY 12Th, 1995.

DUKE UNIVERSITY

DURHAM, NC 27708-0320 